\title{
Canonical bracket in quantum-classical hybrid systems
}

\author{
V. Gil ${ }^{1}$ and L. L. Salcedo ${ }^{1,2}$ \\ ${ }^{1}$ Departamento de Física Atómica, Molecular y Nuclear, Universidad de Granada, E-18071 Granada, Spain \\ ${ }^{2}$ Instituto Carlos I de Física Teórica y Computacional, Universidad de Granada, E-18071 Granada, Spain
}

(Dated: August 3, 2021)

\begin{abstract}
We study compound systems with a classical sector and a quantum sector. Among other consistency conditions we require a canonical structure, that is, a Lie bracket for the dynamical evolution of hybrid observables in the Heisenberg picture, interpolating between the Poisson bracket and the commutator. Weak and strong postulates are proposed. We explicitly construct one such hybrid bracket when the Hilbert space of the quantum sector is finite dimensional and show that it is unique if the strong postulates are enforced. The adjoint bracket for the Schrodinger picture version of the dynamics is also obtained. Unfortunately, preservation of the positivity of the density matrix under the evolution is not guaranteed. The case of a particle with classical position and momentum and quantum spin- $\frac{1}{2}$ is discussed and the spin-orbit dynamics is worked out.
\end{abstract}

\section{Contents}

\section{Introduction}

II. The quantum-classical hybridization problem

A. The space of observables

B. Conditions on the hybrid bracket

C. Alternative hybridization approaches

III. Particle with classical position-momentum and quantum spin- $\frac{1}{2} \quad 9$

A. Hybrid observables and bracket $\quad 9$

B. Hybrid spin-orbit dynamics $\quad 10$

IV. Hybrid Lie bracket $\quad 12$

A. Definition of the bracket $\quad 12$

B. Proof of the Lie bracket property 13

C. Observations

$\begin{array}{ll}\text { V. Uniqueness of the hybrid bracket } & 14\end{array}$

VI. The Schrodinger picture $\quad 17$

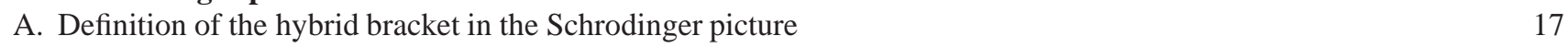

B. Explicit construction of the hybrid bracket in the Schrodinger picture $\quad 18$

C. Classical particle with classical position-momentum and quantum spin- $\frac{1}{2} \quad 19$

$\begin{array}{ll}\text { VII. The positivity fiasco } & 20\end{array}$

A. Heisenberg picture $\quad 20$

B. Schrodinger picture $\quad 21$

\begin{tabular}{ll} 
C. Discussion & 22 \\
\hline
\end{tabular}

\begin{tabular}{lr} 
VIII. Summary and Conclusions & 23 \\
\hline
\end{tabular}

Acknowledgments

A. Mathematical structure of the hybrid bracket $\quad 23$

B. Proof of Eq. (5.19)

\begin{tabular}{ll} 
References & 26 \\
\hline
\end{tabular} 


\section{INTRODUCTION}

The purpose of this note is to consider hypothetical hybrid physical systems involving a truly classical sector in interaction with a truly quantum sector, and analyze the internal consistency of such constructions. There are several motivations in the literature to study those hybrid systems.

One motivation comes from the foundations and interpretation of quantum mechanics. Classical and quantum sectors coexist in the Copenhagen interpretation, where a classical measuring apparatus is postulated [1-4]. A precise formulation of this interpretation seems to require some consistent description of quantum and classical sectors in mutual interaction. Another motivation, also based on the assumption of the existence of true classical systems in nature, comes from Einstein gravity, as some authors have argued that for all we know at present, gravity could be classical. For a discussion see [5-12].

A totally different motivation applies for the more plausible point of view that strictly speaking only quantum systems exist in nature and the classical description is just a macroscopic approximation. Even so, there are many situations where a fully quantum treatment is too difficult to work out and a classical approximation is indicated for some subset of degrees of freedom. In this regard a major impulse to the study of hybrid quantum-classical systems comes from quantum chemistry, molecular physics or condensed matter physics, typically using a quantum description for the fast degrees of freedom and a classical description for the slow ones [13-18]. In this point of view, the quantum-classical system would be intrinsically approximated as a description of nature, yet it would be of interest to have an internally consistent description of it. The situation would be analogous to the problem of solving a set of ordinary differential equations using a numerical method, thereby introducing some error; still one would like to have some properties exactly preserved, as e.g., energy conservation or some exact symmetries [19, 20].

While classical mechanics is not a faithful description of nature, it is nevertheless internally consistent. The case of mathematical consistency is not quite so simple for hybrid quantum-classical systems, except in the trivial case in which the two sectors are never coupled. Prescriptions are needed to specify the hybrid dynamics, and many different approaches can be found in the literature (see [21] for a classification of them). Here we follow the path of previous works [22-25] which keep a classical description for the classical sector and a quantum description for the quantum sector, and emphasize the features that are common to quantum and classical dynamics. Both in classical and quantum dynamics, the observables of compound systems are sums of products of observables of each sector; we assume the same in the hybrid case. Another common feature is the canonical structure. The main goal will be to construct a dynamical bracket for the hybrid system, paralleling the Poisson bracket and the commutator, for the evolution of the observables. As it is well-known these are Lie brackets as this property is needed to implement any canonical dynamics [26], so we seek the same property in the hybrid case. Here we study how far one could go in this direction.

We are guided by the following consideration. Let us consider two classical sectors, each with its own phase space and with dynamics driven by the Poisson bracket in each sector. In this case there is a standard way to combine the two sectors into a new one: the phase space of the compound system is the direct product of spaces (this is a fancy name for the set of linear combinations of products of observables of the two sectors) and the dynamics uses the Poisson bracket of the compound system. Instead of assuming this dynamical bracket for the compound system from the outset, it can be asked whether there are other possibilities. In [25] it is shown that, the (compound) Poisson bracket is the only solution if sufficiently informative constraints are set. The analogous constraints also uniquely select the commutator as the dynamical bracket of the compound system in the case of two quantum sectors. (In passing, the direct product construction guarantees that observables of two different quantum sectors commute.) So for hybrid quantum-classical systems we proceed in a completely similar manner, that is, by taking the space of hybrid observables as the direct product space and postulating conditions common to the classical-classical and the quantum-quantum cases to be fulfilled by the hybrid dynamical bracket.

In Sec. II we give precise definitions of our set of hybrid observables and specify how to obtain expectation values from them. Also we lay down some general requirements to be satisfied by the hybrid dynamical bracket in the Heisenberg picture. The basic requirement being, in addition to a canonical structure, that each sector must evolve according to its own dynamics when the coupling between them is switched off. Other conditions cannot be maintained: Antisymmetry of the bracket already leads to an incompatibility with the naive expectation of the bracket being a derivation, so this latter requirement is dropped. However, the most stringent condition is the requirement of the Jacobi identity, due to its lack of linearity. For instance, considering two quantum sectors and taking their classical limit, one recovers the classical dynamics which certainly fulfills the Jacobi identity, but if the classical limit is taken in just one of the sectors this results in a violation of the identity [25]. (Classical-quantum limits have been studied in [27].) As the minimal postulates are too weak to fully solve for the set of brackets satisfying them, we consider stronger (more restrictive) axioms, still consistent with the correct behavior when the two sectors are decoupled. In the same section we comment on alternative formulations found in the literature which would lead more exotic hybrid scenarios.

In Sec. III we analyze the example of a particle with a classical position and momentum and a quantum spin- $\frac{1}{2}$. We give a precise formulation of such hybrid system showing that rotational invariance is correctly realized on the system. We consider a concrete dynamics of the spin-orbit type and solve explicitly the equations of motion in that case.

In Sec. IV we explictly construct a hybrid dynamical bracket for the particular case of quantum sectors with a finitedimensional Hilbert space. We show that this bracket fulfills the strong postulates laid down in Sec. II and also that it is a 
Lie bracket, enjoying the Jacobi identity property. This is very remarkable as it was proved in [25] that the strong postulates forbid any dynamical Lie bracket when the quantum sector is of position-momentum type [i.e., $\mathscr{H}=L^{2}\left(\mathbb{R}^{n}\right)$ ]. To our knowledge, this is the first instance in the literature of a dynamical hybrid bracket consistent with the Jacobi identity. (We refer only to formulations where hybrid observables are linear combinations of products of observables of each sector. Other formulations do have Lie brackets for the hybrid dynamics, at the price of greatly enlarging the set of admissible observables.)

Sec. $\nabla$ is rather technical and it is entirely devoted to prove that the postulates yield precisely one hybrid bracket when the Hilbert space is finite-dimensional. We constructively determine the most general solution to the postulates and show that the solution is unique. The proof is similar to that in [25], where a position-momentum quantum sector was assumed and a no-go result was derived.

In Sec. VI we discuss the Schrodinger picture for hybrid systems. From the condition that the expectation values must coincide in Heisenberg and Schrodinger pictures, we explicitly construct the adjoint bracket for the dynamics in the Schrodinger picture. As it turns out such bracket is not a Lie bracket nor a derivation.

In Sec. VII we analyze whether the hybrid bracket previously obtained complies with the essential requirement of maintaining the positivity of the density matrix. We find that this is not guaranteed and show counterexamples. This implies that the hybrid dynamics is not physically meaningful, at least for generic hybrid systems. It is not excluded that positivity could be preserved in certain particular cases. This negative result could be a consequence of too strict postulates set on the hybrid dynamics, or perhaps it could simply reflect that hybrid systems cannot be formulated in a consistent manner as exact systems. In that case hybrid dynamics would display intrinsic limitations, remaining as useful approximations to fully quantum systems.

The conclusions are summarized in Sec. VIII In Appendix $\mathrm{A}$ we briefly consider the mathematical structure and possible generalizations. In Appendix B we provide the proof of a lemma needing in Sec. $\nabla$

\section{THE QUANTUM-CLASSICAL HYBRIDIZATION PROBLEM}

\section{A. The space of observables}

Our starting point to formulate hybrid quantum-classical systems is the formal similarity between classical and quantum mechanics within the canonical formalism.

In the canonical formalism, a classical system is described by a phase space with a set of coordinates $x^{i}, k_{i}, i=1, \ldots, n$ and the observables are real functions $A(x, k)$ defined on the phase space. Let us denote $\mathscr{A}_{c}$ the set of classical observables. The dynamical evolution is given by ${ }^{1}$

$$
\frac{d A}{d t}=\{A, H\}
$$

where $H(x, k)$ is the Hamiltonian and $\{$,$\} denotes the Poisson bracket$

$$
\{A, B\}=\sum_{i}\left(\frac{\partial A}{\partial x^{i}} \frac{\partial B}{\partial k_{i}}-\frac{\partial A}{\partial k_{i}} \frac{\partial B}{\partial x^{i}}\right) .
$$

Of course, the classical phase space needs not be of precisely this type, to which we refer as position-momentum type in what follows. For instance, one could work in the subset of observables depending only on the orbital angular momentum $\boldsymbol{L}=\boldsymbol{x} \times \boldsymbol{k}$, i.e., $A=f(\boldsymbol{L})$. These observables close an algebra since the Poisson bracket can be worked out to give

$$
\{A, B\}=\boldsymbol{L} \cdot \frac{\partial A}{\partial \boldsymbol{L}} \times \frac{\partial B}{\partial \boldsymbol{L}},
$$

and $\{A, B\}$ is again a function of $\boldsymbol{L}$. Any set of structure constants of a Lie algebra defines one such Poisson-Lie algebra where the observables are functions of the generators regarded as classical variables.

We do not need to assume a classical sector of position-momentum type in our discussion, for the classical sector it is only necessary that i) the variables commute, the dynamical Poisson bracket be ii) a Lie bracket (i.e., linear, antisymmetric and fulfilling the Jacobi identity) and, iii) a derivation (i.e., obeying the product law). ${ }^{2}$

\footnotetext{
${ }^{1}$ For simplicity we assume observables without explicit time dependence. Otherwise the equation would be $d A / d t=\{A, H\}+\partial A / \partial t$.

2 The word "derivation" is used here in a technical sense. A linear application $D$ is a derivation with respect to a product $*$ if it fulfills the product law: $D(A * B)=D(A) * B+A * D(B)$. Throughout the paper "derivation" refers to the ordinary product, so for instance $D \equiv[A$,$] (where [, ] is the commutator)$ is a derivation: $[A, B C]=[A, B] C+B[A, C]$. Exceptionally in Sec. $\mathrm{V}$ and Appendix $\mathrm{A}$ "derivation" refers to a Lie product. For instance, the Jacobi identity establishes that $D \equiv(A$,$) (being (, ) some Lie product) is a derivation with respect to the product ():,(A,(B, C))=((A, B), C)+(B,(A, C))$.
} 
In the quantum case, the states form a Hilbert space, and the observables correspond to Hermitian operators. Let us denote $\mathscr{A}_{q}$ the set of quantum observables. In the Heisenberg picture they evolve as

$$
\frac{d A}{d t}=\frac{1}{i \hbar}[A, H]=: \llbracket A, H \rrbracket
$$

where $[$,$] denotes the commutator. Like \{$,$\} in \mathscr{A}_{c}$, the operation $\llbracket, \rrbracket$ is also a Lie bracket and a derivation in $\mathscr{A}_{q}$. We assume throughout that $\mathscr{A}_{q}$ (the quantum observables) is the set of all Hermitian operators of the quantum Hilbert space. In Appendix we briefly consider relaxing this condition by demanding only that $\mathscr{A}_{q}$ is a Lie algebra, i.e., linear and closed under commutation, $\llbracket \mathscr{A}_{q}, \mathscr{A}_{q} \rrbracket \subseteq \mathscr{A}_{q}$. (This is necessary if any observable can be part of the Hamiltonian.)

For two classical particles with position and momentum $\left(\boldsymbol{x}_{1}, \boldsymbol{k}_{1}\right)$ and $\left(\boldsymbol{x}_{2}, \boldsymbol{k}_{2}\right)$, the observables of the compound system are functions of $\left(\boldsymbol{x}_{1}, \boldsymbol{k}_{1}, \boldsymbol{x}_{2}, \boldsymbol{k}_{2}\right)$. This is just the tensor product of the sets of observables in both sectors, i.e., $\mathscr{A}_{c}=\mathscr{A}_{c}^{(1)} \otimes \mathscr{A}_{c}^{(2)}$. A similar tensor product occurs in the quantum case for the Hilbert space $\mathscr{H}=\mathscr{H}^{(1)} \otimes \mathscr{H}^{(2)}$ and hence for the set of observables $\mathscr{A}_{q}=\mathscr{A}_{q}^{(1)} \otimes \mathscr{A}_{q}^{(2)}$. For instance $S=S_{1} \otimes \mathbb{1}_{2}+\mathbb{1}_{1} \otimes \boldsymbol{S}_{2}$, for a two spin system. Quite naturally, we will assume a similar construction for a hybrid quantum-classical system. That is, the set of hybrid observables is identified with

$$
\mathscr{A}=\mathscr{A}_{c} \otimes \mathscr{A}_{q} \text {. }
$$

Alternative hybrid constructions in the literature not following this structure are briefly discussed in Sec. IC

According to this definition, the elements of $\mathscr{A}$ are linear combinations of products of c-number functions on the phase space with quantum operators. Equivalently, a hybrid observable $A \in \mathscr{A}$ will be any function defined on the classical phase space taking values on the set of quantum operators $\mathscr{A}_{q}$.

Classical and quantum observables can be regarded as particular types of hybrid observables, so $\mathscr{A}_{c}$ and $\mathscr{A}_{q}$ can be embedded within $\mathscr{A} . \mathscr{A}_{c}$ can be identified with the hybrid observables of the type $C \mathbb{1}$ in $\mathscr{A}$, where $\mathbb{1}$ denotes the identity operator on $\mathscr{H}$, and $C$ is a c-number function of the classical variables. Likewise, $\mathscr{A}_{q}$ can be identified with the $Q \in \mathscr{A}$ which are constant functions with respect to the classical variables. Generic hybrid observables are linear combinations of products $C Q$, with $C \in \mathscr{A}_{c}$ and $Q \in \mathscr{A}_{q}$. The $C$ 's commute with every observable, while $Q$ 's commute with $C$ 's but not with another $Q^{\prime}$ in general. It would seem that the embeddings of $\mathscr{A}_{c}$ and $\mathscr{A}_{q}$ in $\mathscr{A}$ are asymmetric, as one requires being a multiple of $\mathbb{1}$ and the other invokes the requirement of being a constant function. The unifying criterion is that $\llbracket A, \rrbracket \equiv 0$ whenever $A \in \mathscr{A}_{c}$ and $\{A$, $\} \equiv 0$ whenever $A \in \mathscr{A}_{q}$. Constant multiples of $\mathbb{1}$ are simultaneously elements of $\mathscr{A}_{c}$ and $\mathscr{A}_{q}$.

The obvious way to assign expectation values to the hybrids observables is

$$
\langle A\rangle_{\rho}=\int d^{n} x d^{n} k \operatorname{tr}(\rho(x, k) A(x, k)),
$$

where the integral is over the classical phase space and the trace is over the quantum Hilbert space. The density matrix $\rho$ is a positive operator for each $(x, k)$, with normalization $\langle\mathbb{1}\rangle_{\rho}=1$. This form of $\langle A\rangle_{\rho}$ interpolates between the purely classical and the purely quantal cases.

By the same token the hybrid dynamics in $\mathscr{A}$ requires a certain bracket between observables, which will be denoted (, ), so that in the Heisenberg picture

$$
\frac{d A}{d t}=(A, H)
$$

The hybrid Schrodinger picture is discussed in Sec. VI

\section{B. Conditions on the hybrid bracket}

The hybrid dynamical bracket is subject to certain requirements. For instance, if $C$ and $C^{\prime}$ denote classical observables and $Q$ and $Q^{\prime}$ denote quantum ones, one expects ${ }^{3}$

$$
\left(C, C^{\prime}\right)=\left\{C, C^{\prime}\right\}, \quad\left(Q, Q^{\prime}\right)=\llbracket Q, Q^{\prime} \rrbracket, \quad(C, Q)=0, \quad C, C^{\prime} \in \mathscr{A}_{c}, Q, Q^{\prime} \in \mathscr{A}_{q}
$$

This guarantees that when the two sectors are decoupled, that is, in the particular case of a Hamiltonian of the type $H=C+Q$, these sectors behave according to their usual dynamics. These conditions by no means fix the form of $($,$) since generic hybrid$ observables are linear combinations of blocks $C Q$, with $C \in \mathscr{A}_{c}$ and $Q \in \mathscr{A}_{q}$, and a rule for $\left(C Q, C^{\prime} Q^{\prime}\right)$ is needed.

\footnotetext{
${ }^{3}$ As usual in the literature, when there is no possibility of confusion we omit the identity factor $\mathbb{1}$ that would appear, e.g., in $C \mathbb{1}$.
} 
Another obvious requirement is

$$
(A, B)^{\dagger}=(A, B) \quad A, B \in \mathscr{A},
$$

in order to ensure that Hermitian observables remain so under dynamical evolution. Or more generally

$$
(A, B)^{\dagger}=\left(A^{\dagger}, B^{\dagger}\right)
$$

if we include non necessarily Hermitian objects. ${ }^{4}$

Still another obvious requirement is that a constant observable, say $\mathbb{1}$, should not evolve. This would be automatically true if $(,$,$) were a derivation [see Eq. (2.20)]. In that case one would have$

$$
(\mathbb{1}, H)=\left(\mathbb{1}^{2}, H\right)=2(\mathbb{1}, H)=0 .
$$

As we will show $($, ) cannot be a derivation (at variance with $\{$,$\} and \llbracket, \rrbracket$ ), thus we postulate this property:

$$
(A, \mathbb{1})=0 \quad \forall A \in \mathscr{A} .
$$

Of course, these considerations are not new. In [24] a hybrid dynamical bracket was proposed, namely,

$$
(A, B)_{a} \equiv \llbracket A, B \rrbracket+\{A, B\} .
$$

Here $\llbracket A, B \rrbracket$ is the same of Eq. (2.4) considering $A$ and $B$ as operators. Also, $\{A, B\}$ is as defined exactly as in Eq. (2.2), whether $A$ and $B$ are commuting or not. While the bracket in (2.13) is linear and obeys the conditions $[2.8$, it is not antisymmetric. This latter condition is needed to guarantee that, thanks to $(H, H)=0$, a time-independent Hamiltonian is conservative also in the hybrid case. A more promising prescription is [22, 23]

$$
(A, B)_{s} \equiv \llbracket A, B \rrbracket+\frac{1}{2}(\{A, B\}-\{B, A\}) .
$$

This is the hybrid bracket more frequently used in the literature. ${ }^{5}$ It is linear and antisymmetric, however it fails to be a Lie bracket as the Jacobi identity is not fulfilled. See [28-30] for other similar brackets suffering the same problem.

Both the classical and the quantum brackets are Lie brackets, and this is also an essential requirement to have a consistent canonical formalism in the hybrid case. The set of all invertible transformations of a system form a group and the observables act as infinitesimal generators of it. The dynamical evolution is generated by $H$ so that $\delta_{H} A=(-H d t, A)$, but any transformation can be chosen as the evolution. Thus other infinitesimal transformations, such as symmetry groups, are generated through the same bracket

$$
\delta_{G} A=(\delta G, A), \quad \delta G=\sum_{i} \delta a^{i} G_{i}
$$

where the $a^{i}$ are the parameters of the group and the $G_{i}$ are the generators. So for instance a relation

$$
(G, H)=0
$$

is telling simultaneously that $\delta_{H} G=0$ and so $G$ is a constant of motion, and that $\delta_{G} H=0$ and so $G$ generates a symmetry transformation of the dynamics. Hence Noether theorem holds also in the hybrid case. As usual, that $A$ is a constant of motion means that $\langle A\rangle_{\rho}$ in any state $\rho$ is conserved in time.

The Lie group structure of the transformations, and at the infinitesimal level the Lie algebra structure, can only be represented by the action of $\delta_{G}=(\delta G$,$) as an operator in \mathscr{A}$ if $($,$) is a Lie bracket. Specifically, for two infinitesimal transformations$ generated by $\delta_{1} G$ and $\delta_{2} G$, the commutator element ${ }^{6}$ is generated by $\delta_{[1,2]} G=\left(\delta_{1} G, \delta_{2} G\right)$. The group structure demands $\delta_{[1,2]}=\left[\delta_{1}, \delta_{2}\right]$ for any representation of the group [31], and so

$$
\left(\left(\delta_{1} G, \delta_{2} G\right), A\right)=\left(\delta_{1} G,\left(\delta_{2} G, A\right)\right)-\left(\delta_{2} G,\left(\delta_{1} G, A\right)\right) .
$$

\footnotetext{
${ }^{4}$ Genuine hybrid observables are Hermitian, however, for convenience we often extend the concept of observable to include complex functions and non Hermitian operators (more precisely, operators of the type $A+i B$ with $A$ and $B$ Hermitian).

${ }^{5}$ Actually, this bracket was introduced to describe the evolution of the density matrix, $d \rho / d t=(H, \rho)_{s}$, in the Schrodinger picture. The new insight of [24] was to introduce the hybrid bracket in the Heisenberg picture.

${ }^{6}$ For two elements $g_{1}, g_{2}$ of a group, its commutator $g_{[1,2]}$ is defined as $g_{1}^{-1} g_{2}^{-1} g_{1} g_{2}$.
} 
This is equivalent to the Jacobi identity, on account of the antisymmetry.

An immediate consequence of the Jacobi identity is that if the relation $C=(A, B)$ holds at time $t=0, C(t)=(A(t), B(t))$ holds at any time $t$, where $A(t)$ represents the Heisenberg picture evolution of $A$ and similarly for $B$ and $C$. More generally, $\delta_{G}=(G$, is an infinitesimal canonical transformation which preserves the canonical relations between the canonical variables. Also, if $A$ and $B$ are constants of motion, $(A, B)$ is again a constant of motion, thanks to the Jacobi identity.

Some times the generators of a group of transformations acting in a tensor space are just the sum of generators in each factor space. For instance $\boldsymbol{J}=\boldsymbol{L}+\boldsymbol{S}$ in $\mathscr{H}=L^{2}\left(\mathbb{R}^{3}\right) \otimes \mathbb{C}^{2}$ for a quantum particle with spin $1 / 2$. The orbital part generates the rotations in $L^{2}\left(\mathbb{R}^{3}\right)$ and $\boldsymbol{S}=\hbar \boldsymbol{\sigma} / 2$ generates the rotations in $\mathbb{C}^{2}$. For $\mathscr{A}=\mathscr{A}_{c} \otimes \mathscr{A}_{q}$, one can consider generators of the type

$$
G_{i}=C_{i}+Q_{i}, \quad C_{i} \in \mathscr{A}_{c}, \quad Q_{i} \in \mathscr{A}_{q}
$$

In this case, the conditions in Eq. (2.8) are already sufficient to guarantee that $G_{i}$ reproduces the Lie algebra of the group,

$$
\left(G_{i}, G_{j}\right)=c_{i j}{ }^{k} G_{k},
$$

(where $c_{i j}{ }^{k}$ are the structure constants) if $C_{i}$ and $Q_{i}$ fulfill the analogous relations. Unfortunately, the tensor product (or rather tensor sum) form in Eq. (2.18) is not sufficiently general. It is good enough for some kinematical symmetry transformations, such as translations and rotations, or some internal symmetries, but it already fails for the Poincare group, which contains the boosts and the Hamiltonian within the generators. The Hamiltonian takes the tensor sum form only in the rather trivial case of no interaction between the classical and quantum sectors, and the same holds for the boost generators.

Another question is whether the dynamical bracket should be a derivation, that is, should fulfill Leibniz law, ${ }^{7}$

$$
(A, B C)=(A, B) C+B(A, C) .
$$

In fact the product law leads to a contradiction with linearity, antisymmetry and $(C, Q)=0$ [32]. Consider for instance a hybrid system with classical orbital angular momentum $\boldsymbol{L}$ and quantum $\operatorname{spin} \boldsymbol{S}$, then

$$
0=(\boldsymbol{L} \cdot \boldsymbol{S}, \boldsymbol{L} \cdot \boldsymbol{S})=\left(L_{i} S_{i}, L_{j}\right) S_{j}+L_{j}\left(L_{i} S_{i}, S_{j}\right)=\varepsilon_{i j k} L_{k} S_{i} S_{j}+L_{j} L_{i} \varepsilon_{i j k} S_{k}=i \hbar \boldsymbol{L} \cdot \boldsymbol{S} .
$$

In the second equation we have used that $\boldsymbol{L}$ and $\boldsymbol{S}$ are angular momenta $\left[\left(L_{i}, L_{j}\right)=\varepsilon_{i j k} L_{k}\right.$, and similarly $\left.S_{i}\right]$ and in the last equality it has been used that $\boldsymbol{S} \times \boldsymbol{S}=i \hbar \boldsymbol{S}$ but $\boldsymbol{L} \times \boldsymbol{L}=0$ classically, since the $L_{i}$ are commuting variables.

More generally, if there are two sectors such that $(C, Q)=0$ and $C$ 's commute with $Q$ 's, and a bracket $($,$) is a two-sided$ derivation, two different expansions hold

$$
\begin{aligned}
& \left(C Q, C^{\prime} Q^{\prime}\right)=\left(C Q, C^{\prime}\right) Q^{\prime}+C^{\prime}\left(C Q, Q^{\prime}\right)=\left(C, C^{\prime}\right) Q Q^{\prime}+C^{\prime} C\left(Q, Q^{\prime}\right), \\
& \left(C Q, C^{\prime} Q^{\prime}\right)=\left(C, C^{\prime} Q^{\prime}\right) Q+C\left(Q, C^{\prime} Q^{\prime}\right)=\left(C, C^{\prime}\right) Q^{\prime} Q+C C^{\prime}\left(Q, Q^{\prime}\right) .
\end{aligned}
$$

We can see that there is a problem if the $C$ 's are c-numbers and the $Q$ 's are not. More explicitly, subtracting the two expressions one finds

$$
\left(C, C^{\prime}\right)\left[Q, Q^{\prime}\right]=\left[C, C^{\prime}\right]\left(Q, Q^{\prime}\right) .
$$

This implies that such bracket is only consistent in two cases: i) all the variables commute, i.e., both sectors are classical, or ii) $()=,[] /,(i \hbar)$ for some $\hbar$ common to the two sectors, i.e., both sectors are quantal. The uniqueness of $\hbar$ has been noted in [32-34].

Therefore a hybrid quantum-classical bracket cannot be a derivation. The product law would guarantee that, if $C=A B$ at $t=0, C(t)=A(t) B(t)$ at any other time. The failure to comply with this implies that classical variables can evolve into non commuting observables, in general. ${ }^{8}$ This is the way quantum fluctuations propagate into the classical sector, the so-called quantum back-reaction. In fact, as shown in [25], quantum fluctuations are not allowed for degrees of freedom which are purely c-number variables.

It should be noted that the product of observables has a physical meaning when the two observables commute with each other, namely, $A B$ represents the product of the numerical values of $A$ and $B$. This is the case of two classical variables or of two observables belonging to two different sectors. No such a physical meaning is attached to the product when the observables

\footnotetext{
${ }^{7}$ Hence also $(A B, C)=A(B, C)+(A, C) B$ due to antisymmetry.

${ }^{8}$ In [32] it is argued that if observables of two sectors commute at $t=0$ but not at other times this implies an unacceptable privileged origin of time. In fact this is only so if the product is regarded as a privileged operation too: one can evolve the product at $t=0$ into a new isomorphic $*$-product at time $t$ and observables would commute with respect to this new product. Going even further, one can change the representation of the observables so that in the new representation the $*$-product is just the ordinary product. This is the Schrodinger picture.
} 
do not commute. Actually, the product of two non-commuting quantum Hermitian operators is not Hermitian, and so is not an observable. ${ }^{9}$

The axioms in Eqs. 2.8, 2.9 and 2.12), plus the Lie bracket condition are basic requirements on the hybrid dynamical bracket. A further requirement is that positivity of a hybrid observable must be preserved by the evolution. This is the case in quantum and classical dynamics. We postpone the discussion of this requirement which is deferred to Sec. VII

Regrettably the postulates in 2.8 are two general (too weak) to be able to obtain the most general bracket consistent with them. So, to be able to obtain definite answers, we will replace 2.8 with stronger assumptions. Namely, following [25] we will require

$$
(C, A)=\{C, A\}, \quad(Q, A)=\llbracket Q, A \rrbracket, \quad \forall C \in \mathscr{A}_{c}, Q \in \mathscr{A}_{q}, A \in \mathscr{A},
$$

or equivalently

$$
\left(C, C^{\prime} Q^{\prime}\right)=\left\{C, C^{\prime}\right\} Q^{\prime}, \quad\left(Q, C^{\prime} Q^{\prime}\right)=\llbracket Q, Q^{\prime} \rrbracket C^{\prime}, \quad C, C^{\prime} \in \mathscr{A}_{c}, Q, Q^{\prime} \in \mathscr{A}_{q}
$$

Clearly these postulates imply those in Eq. 2.8. They also guarantee Eq. (2.12), since $\mathbb{1}$ is simultaneously a purely classical and a purely quantal observable.

The justification of the stronger postulates is as follows: They are such that the observable $C^{\prime} Q^{\prime}$ evolves into $C^{\prime}(t) Q^{\prime}$ if $H=C$, and to $C^{\prime} Q^{\prime}(t)$ if $H=Q$. That is, if the two sectors are dynamically uncoupled (i.e., $H=C+Q$ ) each factor in a product observable $C^{\prime} Q^{\prime}$ evolves independently, according to its own dynamics. This property certainly holds true for two classical sectors or for two quantum sectors, so we postulate the same property in the hybrid case.

The axioms (2.24) immediately imply that the operators of the type $(C$,$) and (Q$,$) , and hence also (C+Q$,$) , act as derivations$ in $\mathscr{A}$. (This property will be used in Sec. III)

On the other hand, $(A$,$) is a derivation on \mathscr{A}_{c}$ or $\mathscr{A}_{q}$ (but not in $\mathscr{A}$ in general), since

$$
\left(A, C C^{\prime}\right)=(A, C) C^{\prime}+C\left(A, C^{\prime}\right), \quad\left(A, Q Q^{\prime}\right)=(A, Q) Q^{\prime}+Q\left(A, Q^{\prime}\right)
$$

An immediate consequence of this latter property is that when one or more classical observables $C_{i}$ are constants of motion (i.e., $\left.\left(H, C_{i}\right)=0\right)$ any function of them is also conserved. The same is true for functions of quantum conserved observables. Nothing would be implied, in principle, for the conservation of products of two arbitrary (hybrid) constants of motion. Nevertheless, we show below that the product of a classical constant of motion with a hybrid constant of motion is indeed conserved [point 7) at the end of Sec. IV C].

The axioms (2.24) do not directly stipulate any condition for $\left(C Q, C^{\prime} Q^{\prime}\right)$, however, the Lie bracket requirement is rather stringent. In fact, it is shown in [25] that there is no Lie bracket fulfilling 2.24 when both the classical and the quantum sectors are of position-momentum type. The proof is made explicitly for the one dimensional case but it holds for any number of dimensions. Also, the precise nature of the classical sector is not relevant. This implies that a fully consistent formulation of hybrid quantum-classical systems with quantum sector $\mathscr{H}=L^{2}\left(\mathbb{R}^{n}\right)$ does not exist, at least if one insist on the stronger postulates 2.24.

What we show below is that if the Hilbert space of the quantum sector is finite-dimensional, it is possible to construct a quantum-classical Lie bracket obeying the axioms (2.24) plus 2.9), and moreover, such bracket is unique. ${ }^{10}$ Unfortunately, we also show in Sec. VII that the hybrid bracket does not preserve positivity, and so it is not acceptable as a consistent formulation.

\section{Alternative hybridization approaches}

Before leaving this section, we want to mention other approaches used in the literature to define quantum-classical hybrid systems.

The traditional approach to mixing quantum and classical degrees of freedom is through a mean-field or Ehrenfest dynamics. In such dynamics there is a Hamiltonian $H(x, k)$ which is an Hermitian operator in the quantum Hilbert space and a function of the classical variables, so it is a hybrid observable of $\mathscr{A}=\mathscr{A}_{c} \otimes \mathscr{A}_{q}$. The quantum sector evolves following the Schrodinger equation, and there the classical degrees of freedom act as time-dependent external parameters appearing in the quantum Hamiltonian. On

\footnotetext{
${ }^{9}$ In some hybridization schemes the concept of product of two arbitrary hybrid observables is simply never introduced nor required. On the other hand a meaning can be given to the commutator $[A, B]$, since $\left\langle-i\left[A\left(t_{1}\right), B\left(t_{2}\right)\right]\right\rangle\left(t_{1}>t_{2}\right)$ is the perturbation on $\left\langle A\left(t_{1}\right)\right\rangle$ due to a small term $B$ added to the Hamiltonian at time $t_{2}$ [35].

${ }^{10}$ At least if one insist that $\mathscr{A}_{q}$ is the set of all operators.
} 
the other hand the classical degrees of freedom evolve classically using as classical Hamiltonian the expectation value of the hybrid Hamiltonian in the instantaneous quantum state. That is,

$$
i \hbar \frac{d \psi}{d t}=H(x, k) \psi, \quad \frac{d x^{i}}{d t}=\frac{\partial\langle H(x, k)\rangle_{\psi}}{\partial k_{i}}, \quad \frac{d k_{i}}{d t}=-\frac{\partial\langle H(x, k)\rangle_{\psi}}{\partial x^{i}} .
$$

In this dynamics the two sectors affect each other but the classical variables remain classical as they do not inherit any quantum fluctuations, hence the so-called quantum back-reaction is missed [36, 37].

Recently it has been emphasized that the mean-field dynamics can be expressed in the form of a Lie bracket between hybrid observables [38-40]. The observables are represented by functions of $x^{i}$ and $k_{i}$ as well as $\psi_{\alpha}$ and $i \hbar \psi_{\alpha}^{*}$, where $|\psi\rangle=\sum_{\alpha} \psi_{\alpha}|\alpha\rangle$. For instance, any quantum operator $Q$ is represented by $\langle\psi|Q| \psi\rangle$, and the Hamiltonian by $\langle\psi|H(x, k)| \psi\rangle$, while a classical observable $C$ is represented by $C\langle\psi \mid \psi\rangle$. The normalization $\langle\psi \mid \psi\rangle=1$ is preserved by the dynamics. The dynamical bracket is the Poisson bracket taking as $\psi_{\alpha}$ and $i \hbar \psi_{\alpha}^{*}$ as canonical conjugated variables, in addition to $x^{i}$ and $k_{i}$. The dynamics is generated by the Poisson bracket between the Hamiltonian and the observables, as in Eq. 2.1. From this point of view, the canonical Ehrenfest dynamics has a proper Lie bracket and also preserves the usual classical and quantum dynamics when the two sectors are not coupled in the Hamiltonian.

There are several well-known problems with the mean field approach (see e.g., [23, 37, 41]). Here we highlight two of them.

The first problem is that initially one starts with observables of the type $\langle\psi|A| \psi\rangle$ with $A \in \mathscr{A}_{=} \mathscr{A}_{c} \otimes \mathscr{A}_{q}$, but the Poisson bracket [using $(x, k)$ and $\left(\psi, i \hbar \psi^{*}\right)$ as classical variables] does not preserve this form (is not the expectation value of any operator). So $(\mathscr{A}, \mathscr{A}) \nsubseteq \mathscr{A}$ and $\mathscr{A}$ does not close a Lie algebra of observables. The minimal algebra containing $\mathscr{A}$ is very large, it probably contains all kinds of functionals $F\left(x, k, \psi, i \hbar \psi^{*}\right)$ subjected only to the condition of invariance under $\left(\psi, i \hbar \psi^{*}\right) \rightarrow\left(\omega \psi, \omega^{*} i \hbar \psi^{*}\right)$ with $\omega \in \mathrm{U}(1)$, that is, arbitrary functions of the blocks $\psi_{\alpha}^{*} \psi_{\beta}$. As argued above, in classical or in quantum mechanics, when two different sectors are coupled, the observables are linear combinations of products of the two sectors. The canonical Ehrenfest dynamics is qualitatively different in this regard, as it prompts an explosion in the number of new hybrid observables. This is one example of emergent phenomenon that would only arise when a classical and a quantum sector are coupled.

The second problem is related to statistical consistency, as noted in [42]: Let us assume that initially the two sectors are decoupled and the hybrid state is a certain density matrix $\rho$ in the quantum sector and some definite state in the classical sector (this is not important), then let us switch on the coupling between both sectors. In order to apply the mean-field dynamics one can proceed by decomposing $\rho$ as a statistical mixture of (not necessarily orthogonal) pure states, that is,

$$
\rho=\sum_{n} p_{n}\left|\varphi_{n}\right\rangle\left\langle\varphi_{n}\right|, \quad\left\langle\varphi_{n} \mid \varphi_{n}\right\rangle=1, \quad p_{n}>0, \quad \sum_{n} p_{n}=1
$$

Then one can apply the mean-field evolution for each such pure state $\left|\varphi_{n}\right\rangle$ coupled to the classical sector. By definition of statistical mixture, the expectation values will be obtained by averaging over the various histories (one for each pure state $\left|\varphi_{n}\right\rangle$ ) with weight $p_{n}$. However, the decomposition of a density matrix is not unique. As it turns out [42] the averages of the hybrid observables depend not only on $\rho$ but also on the concrete decomposition adopted. For instance, a beam of unpolarized electrons (a quantum state) can be obtained by an equal mixture of spin up and down electrons with respect to any given quantization axis $\hat{n}$. According to the mean field dynamics, if the beam passes through a chunk of classical material the evolution will depend on the concrete choice of axis $\hat{n}$. This would be another emergent phenomenon: even if quantum mechanically two systems with the same density matrix are completely indistinguishable, the coupling to the classical sector would uncover hidden information in the mixture: the experiment would be able to distinguish between different mixtures of pure states leading to the same density matrix. Note that the presence of hidden information would be a feature of ordinary quantum mechanics, even if it is only uncovered through coupling to a classical sector. However, if all those new variables encoding the hidden information in $\rho$ exists (in ordinary quantum mechanics) one can ask whether they would not contribute to the entropy (since they encode physical information) and get thermalized, as any other degree of freedom, and reflect on the observed specific heat, for instance.

We find this very problematic. At variance with [21] we propose here a principle of non (strong) emergence in quantumclassical hybrid systems. If there were any truly classical systems in nature they would be the measuring apparatuses of the Copenhagen interpretation. But it is precisely through experiments that the standard quantum mechanics is established, thus any quantum-classical hybrid approach implying extraordinary effects uncovered when quantum systems are coupled to (hypothetical) classical ones should be rejected, in our view.

Of course an easy way out would seem to express the mean field prescription using a density matrix $\rho_{q}$ for the quantum sector:

$$
i \hbar \frac{d \rho_{q}}{d t}=\left[H(x, k), \rho_{q}\right], \quad \frac{d x^{i}}{d t}=\frac{\partial\langle H(x, k)\rangle_{\rho_{q}}}{\partial k_{i}}, \quad \frac{d k_{i}}{d t}=-\frac{\partial\langle H(x, k)\rangle \rho_{q}}{\partial x^{i}} .
$$

However, even when a hybridization prescription is formulated using directly $\rho_{q}$ (instead of pure states), statistical consistency is not automatically guaranteed. By definition of mixture, if $\rho_{q}=p_{1} \rho_{1}+p_{2} \rho_{2}$, we should demand for the expectation values 
that $\langle A\rangle_{\rho_{q}}=p_{1}\langle A\rangle_{\rho_{1}}+p_{2}\langle A\rangle_{\rho_{2}}$. Essentially this requires linearity in $\rho_{q}$ in the evolution equations, and this requirement is not fulfilled in Eq. (2.29).

Linearity holds true by construction in the hybrid Heisenberg picture described above, Eq. 2.7). When the evolution of observables is transferred to $\rho$, through Eq. 2.6), one obtains in the Schrodinger picture

$$
\frac{d \rho}{d t}=(H, \rho)^{\prime},
$$

where $(,)^{\prime}$ is the adjoint of $($,$) (see Sec. VI). An equation like (2.30) cannot be written for the mean-field evolution.$

It is easy to see that the two problems of explosion in the number of hybrid observables and loss of statistical consistency are related and are intrinsic to any mean-field-like dynamics. The idea behind the Ehrenfest formulation is to describe the two sectors in the classical setting of the phase space $\mathscr{M}$ with coordinates $\boldsymbol{X}=\left(\boldsymbol{x}, \boldsymbol{k}, \boldsymbol{\psi}, \boldsymbol{\psi}^{*}\right)$, where $\boldsymbol{\psi}$ is the vector with components $\psi_{\alpha}$ (if desired one can use real vectors $\operatorname{Re} \boldsymbol{\psi}$ and $\operatorname{Im} \boldsymbol{\psi}$ as coordinates, this is unessential). In that dynamics each point $\boldsymbol{X}$ is a configuration of the hybrid system and it evolves under a certain classical flow describing an orbit $\boldsymbol{X}(t)$ on $\mathscr{M}$. A mixed state corresponds to a probability density $\sigma(\boldsymbol{X})$ on $\mathscr{M}$. If initially $\sigma$ is Dirac delta at some point $\boldsymbol{X}$, it will be a Dirac delta at $\boldsymbol{X}(t)$ at time $t$, so in particular the marginal density $\sigma(\boldsymbol{x}, \boldsymbol{k})$ will also be a Dirac delta at all times: no fuzziness in the classical variables is inherited by their coupling to the quantum sector, no quantum back-reaction. The hybrid observables are functions $F(\boldsymbol{X})$ on $\mathscr{M}$ which evolve as $F(\boldsymbol{X}(t))$. The subset of observables in $\mathscr{A}$ corresponds to functions which are bilinear in $\boldsymbol{\psi}$ and $\boldsymbol{\psi}^{*}$, that is $A_{\alpha \beta}(\boldsymbol{x}, \boldsymbol{k}) \psi_{\alpha}^{*} \psi_{\beta}$. If one goes outside this set, new observables emerge and also statistical consistency is lost. The problem is that in all interesting cases $(\boldsymbol{x}(t), \boldsymbol{k}(t))$ will depend on the initial $\left(\boldsymbol{\psi}, \boldsymbol{\psi}^{*}\right)$ and this additional dependence implies a breaking in the bilinearity on $\left(\boldsymbol{\psi}, \boldsymbol{\psi}^{*}\right)$ in the observable. The problem would be avoided if $(\boldsymbol{x}(t), \boldsymbol{k}(t))$ did not depend on the quantum sector but this does not really describe two sectors in mutual interaction. The problem noted is very general, even if the concrete flow of the mean-field dynamics were replaced by something more sophisticated.

We could cast our own hybrid systems using the extended phase space $\mathscr{M}$ and observables as functions of the bilinear type $A_{\alpha \beta}(\boldsymbol{x}, \boldsymbol{k}) \psi_{\alpha}^{*} \psi_{\beta}$ on it. We seek a suitable Lie bracket defined on that set of functions but do not require from the outset that $A(X ; t)=A(X(t))$, which as we have just noted, would break the bilinearity condition.

There is a whole variety of hybridization prescriptions in the literature, such as that based on the Hamilton-Jacobi approach [43, 44], the mapping of the classical sector into a quantum one [45, 46], or the Bohmian particle [47 49], and many other (see e.g. [36, 50]), each with its own consistency problems. Some of them are summarized in [21] and criticized in [25, 42, 51]. Here we stick to the Heisenberg picture approach based on $\mathscr{A}_{c} \otimes \mathscr{A}_{q}$ with a canonical formulation based on a dynamical Lie bracket, as it seems to be closest to a standard purely quantum or purely classical description. This approach bypasses the problem of explosion of hybrid observables, the emergence of extraordinary effects uncovered by the hybrid coupling or problems with statistical consistency. The one solution we find is flawed, however, by the lack of positivity of the measure, a fatal problem already observed long ago in [23] for the bracket $(,)_{s}$.

\section{PARTICLE WITH CLASSICAL POSITION-MOMENTUM AND QUANTUM SPIN- $\frac{1}{2}$}

In this section we consider the hybrid system of a particle with classical position and momentum and quantum spin- $\frac{1}{2}$. Versions of this problem have been considered before in the literature. For instance, Ref. [52] makes one such study in the context of a Hamilton-Jacobi formulation, which is subject to the criticism expressed in Sec. IIC.

\section{A. Hybrid observables and bracket}

The observables of the classical sector are complex-valued functions $f(\boldsymbol{x}, \boldsymbol{k})$. This defines the set $\mathscr{A}_{c}$. On the other hand, the quantum Hilbert space is $\mathbb{C}^{2}$, so the quantum operators are $2 \times 2$ complex matrices. This defines $\mathscr{A}_{q}$. These can be expressed as $\sum_{\mu=0}^{3} a_{\mu} \sigma_{\mu}$ where the $a_{\mu}$ are four complex numbers, $\sigma_{i}, i=1,2,3$ are the Pauli matrices and $\sigma_{0}=\mathbb{1}$ is the $2 \times 2$ identity matrix.

The hybrid quantum-classical observables are generated from tensor product of the quantum and classical sector, so they are linear combinations of products of functions of $(\boldsymbol{x}, \boldsymbol{k})$ with the $\sigma_{\mu}$. This defines $\mathscr{A}=\mathscr{A}_{c} \otimes \mathscr{A}_{q}$. Therefore, the hybrid observables are functions of $\boldsymbol{x}$ and $\boldsymbol{k}$ that take values in the set of $2 \times 2$ matrices,

$$
A \in \mathscr{A} \quad A(\boldsymbol{x}, \boldsymbol{k})=\left(\begin{array}{cc}
A_{0}+\frac{\hbar}{2} A_{3} & \frac{\hbar}{2} A_{1}-\frac{i \hbar}{2} A_{2} \\
\frac{\hbar}{2} A_{1}+\frac{i \hbar}{2} A_{2} & A_{0}-\frac{\hbar}{2} A_{3}
\end{array}\right)=A_{0}(\boldsymbol{x}, \boldsymbol{k}) \mathbb{1}+\boldsymbol{A}(\boldsymbol{x}, \boldsymbol{k}) \cdot \boldsymbol{S}, \quad \boldsymbol{S} \equiv \frac{\hbar}{2} \boldsymbol{\sigma} .
$$

For proper observables $A=A^{\dagger}$ and the functions $A_{\mu}(\boldsymbol{x}, \boldsymbol{k})$ are real.

A hybrid observable $A$ is purely classical when it acts as a multiple of the identity on the quantum sector, i.e., when $A=A_{0} \mathbb{1}$ and $\boldsymbol{A} \equiv 0$. Such observable commutes with any other observable, that is, $\llbracket A, \rrbracket \equiv 0$. On the other hand, $A$ is purely quantal when 
the four functions $A_{\mu}(\boldsymbol{x}, \boldsymbol{k})$ are actually independent of $(\boldsymbol{x}, \boldsymbol{k})$ i.e., take a constant value $a_{\mu}$ on the phase space. For such a purely quantal observable $\{A,\} \equiv 0$.

For a hybrid observable $A$, we will call $A_{0}$ the classical part of the observable and $\boldsymbol{A} \cdot \boldsymbol{S}$ its quantal part.

To assign a canonical structure in the hybrid system, we need to define a Lie bracket in the set of hybrid observables. A Lie bracket that fulfills the axioms in Eq. 2.24), as well as 2.9), is the following one

$$
\left(A_{0}+\boldsymbol{A} \cdot \boldsymbol{S}, B_{0}+\boldsymbol{B} \cdot \boldsymbol{S}\right):=\left\{A_{0}, B_{0}\right\}+\left\{A_{0}, \boldsymbol{B}\right\} \cdot \boldsymbol{S}+\left\{\boldsymbol{A}, B_{0}\right\} \cdot \boldsymbol{S}+\boldsymbol{A} \times \boldsymbol{B} \cdot \boldsymbol{S}
$$

It is easy to see that this bracket fulfills the axioms 2.24). Indeed, when $B=C \mathbb{1}$ is purely classical

$$
(A, C)=\left(A_{0}+\boldsymbol{A} \cdot \boldsymbol{S}, C\right)=\left\{A_{0}, C\right\}+\{\boldsymbol{A}, C\} \cdot \boldsymbol{S}=\{A, C\},
$$

Likewise, when $Q=Q_{0} \mathbb{1}+Q \cdot S$ is purely quantal [the $Q_{\mu}$ are constant with respect to $(x, k)$ ]

$$
(A, Q)=\left(A_{0}+\boldsymbol{A} \cdot \boldsymbol{S}, Q_{0}+\boldsymbol{Q} \cdot \boldsymbol{S}\right)=\boldsymbol{A} \times \boldsymbol{Q} \cdot \boldsymbol{S}=\llbracket A, Q \rrbracket .
$$

Obviously the bracket in Eq. (3.2) is also linear and antisymmetric. Remarkably, it obeys the Jacobi identity too. This will follow as a particular case in next section. In fact this is the unique Lie bracket consistent with the postulates. This is shown in Sec. D

The total angular momentum, with the usual form,

$$
\boldsymbol{J}=L+S, \quad L=x \times k
$$

fulfills the angular momentum algebra, $\left(J_{i}, J_{j}\right)=i \varepsilon_{i j k} J_{k}$, and is the generator of the rotations in $\mathscr{A}$. As we noted in the previous section, the postulates 2.24) guarantee that operators of the form $(C+Q$,$) are derivations. So (\boldsymbol{J}$,$) is a derivation and this$ is useful to construct observables which are scalar under rotations, or more generally, tensor with well defined transformations under rotations. For instance, $\boldsymbol{k}$ and $\boldsymbol{S}$ are vectors and $\boldsymbol{k} \cdot \boldsymbol{S}$ is a scalar, i.e.,

$$
\left(J_{i}, k_{j}\right)=\varepsilon_{i j k} k_{k}, \quad\left(J_{i}, S_{j}\right)=\varepsilon_{i j k} S_{k}, \quad(\boldsymbol{J}, \boldsymbol{k} \cdot \boldsymbol{S})=0 .
$$

In general, a hybrid observable constructed with the basic vectors $\boldsymbol{x}, \boldsymbol{k}$, and $\boldsymbol{S}$ plus $\delta_{i j}$ and $\varepsilon_{i j k}$, as usual, has the standard tensorial behavior under rotations. This follows from the fact that $\boldsymbol{L}$ in $\boldsymbol{J}$ produces the usual rotation on the classical factors and $\boldsymbol{S}$ on the quantum factors of the observable, while $\delta_{i j}$ and $\varepsilon_{i j k}$ are unchanged due to $(\boldsymbol{J}, \mathbb{1}) \equiv 0$.

\section{B. Hybrid spin-orbit dynamics}

Let us consider a concrete dynamics for the particle with classical position-momentum and quantum spin- $\frac{1}{2}$. For instance let

$$
H=\frac{\boldsymbol{k}^{2}}{2 M}+g \boldsymbol{L} \cdot \boldsymbol{S} .
$$

In this dynamics, the total angular momentum is conserved

$$
(\boldsymbol{J}, H)=0,
$$

and in turn, $H$ is a scalar under rotations. Other constants of motion are $\boldsymbol{k}^{2}, \boldsymbol{L}^{2}, \boldsymbol{L} \cdot \boldsymbol{S}$, and $\boldsymbol{S}^{2}$ as is readily verified (in fact, $\left.S^{2}=\frac{3}{4} \hbar^{2} \mathbb{1}\right)$

The classical orbital angular momentum and the quantum spin are not separately conserved,

$$
(\boldsymbol{L}, H)=-(\boldsymbol{S}, H)=-g \boldsymbol{L} \times \boldsymbol{S} .
$$

This gives their variation to $O(t)$. The full evolution of $\boldsymbol{S}$ is given by

$$
S(t)=R_{t}^{-1} S
$$

where $R_{t}$ is the rotation with parameters $\phi=-g t \boldsymbol{L} .{ }^{11}$ Correspondingly

$$
\boldsymbol{L}(t)=\boldsymbol{J}-\boldsymbol{S}(t)=\boldsymbol{L}-\left(R_{t}^{-1}-1\right) \boldsymbol{S},
$$

\footnotetext{
${ }^{11}$ For $\phi=\phi \hat{n}$, with $\hat{n}^{2}=1, \hat{n}$ is the rotation axis and $\phi$ the rotation angle.
} 
So $L(t)$ picks up a quantum component during its evolution. Note that because the bracket is not a derivation $(A B)(t)$ needs not coincide with $A(t) B(t)$. In particular $\left(\boldsymbol{L}^{2}\right)(t)=\boldsymbol{L}^{2}$ does not coincide with $\boldsymbol{L}(t)^{2}$. Nevertheless $\boldsymbol{L}^{2}$ is a constant of motion and this is still a meaningful non trivial statement, namely, the expectation value $\left\langle\boldsymbol{L}^{2}\right\rangle_{\rho(t)}$ remains time-independent in any state as $\rho(t)$ evolves in the Schrodinger picture.

Also, let us note that, even if $\dot{\boldsymbol{L}}=(\boldsymbol{L}, H)=-g \boldsymbol{L} \times \boldsymbol{S}$ (at $t=0$ ), this does not imply that $\ddot{\boldsymbol{L}}$ is given by $-g(\dot{\boldsymbol{L}} \times \boldsymbol{S}+\boldsymbol{L} \times \dot{\boldsymbol{S}}$ ), as the product law does not apply. Actually, that expression is not even Hermitian. The correct result is instead

$$
\ddot{\boldsymbol{L}}=((\boldsymbol{L}, H), H)=g^{2}\left(\boldsymbol{L}^{2} \boldsymbol{S}-\boldsymbol{L} \cdot \boldsymbol{S} \boldsymbol{L}\right) .
$$

The full evolution of a generic observable can be worked out for $H=g \boldsymbol{L} \cdot \boldsymbol{S}$, i.e., in the large $M$ limit. (Although in this case the coupling $g$ can be eliminated by rescaling the time, we keep it for clarity.) Let

$$
A(\boldsymbol{x}, \boldsymbol{k} ; t)=a(\boldsymbol{x}, \boldsymbol{k} ; t)+\boldsymbol{b}(\boldsymbol{x}, \boldsymbol{k} ; t) \cdot \boldsymbol{S} .
$$

The equations of motion (2.7) can be written as

$$
\frac{\partial a}{\partial t}=0, \quad \frac{\partial \boldsymbol{b}}{\partial t}=g\{a, \boldsymbol{L}\}+g \boldsymbol{b} \times \boldsymbol{L} .
$$

Note that here $\boldsymbol{x}$ and $\boldsymbol{k}$, or $\boldsymbol{L} \equiv \boldsymbol{x} \times \boldsymbol{k}$, are just variables, they do not evolve. The evolution is on $a$ and $b$.

The first equation says that actually $a$ is time-independent. Taking a new time derivative gives

$$
\frac{\partial^{2} \boldsymbol{b}}{\partial t^{2}}=g \frac{\partial \boldsymbol{b}}{\partial t} \times \boldsymbol{L}
$$

with solution

$$
\frac{\partial \boldsymbol{b}}{\partial t}=\left.R_{t} \frac{\partial \boldsymbol{b}}{\partial t}\right|_{t=0},
$$

where $R_{t}$ the same as in Eq. 3.10 (i.e., a rotation of angle $-g t L$ over the axis $L / L$ ). Thus the full solution is

$$
\begin{aligned}
& a(t)=a(0), \\
& \boldsymbol{b}(t)=R_{t} \boldsymbol{b}(0)+g t \frac{\boldsymbol{h} \cdot \boldsymbol{L}}{L^{2}} \boldsymbol{L}+\frac{1}{L^{2}}\left(R_{t}-1\right)(\boldsymbol{L} \times \boldsymbol{h}),
\end{aligned}
$$

where $L=|\boldsymbol{L}|$ and

$$
\boldsymbol{h} \equiv\{a, \boldsymbol{L}\}=\boldsymbol{x} \times \frac{\partial a}{\partial \boldsymbol{x}}+\boldsymbol{k} \times \frac{\partial a}{\partial \boldsymbol{k}} .
$$

For $A(0)=\boldsymbol{S}$ this formula reproduces Eq. (3.10), and Eq. 3.11) is reproduced too. For $A(0)=\boldsymbol{x}$ one obtains

$$
x(t)=x+g t \boldsymbol{L} \times x \frac{\boldsymbol{L} \cdot \boldsymbol{S}}{L^{2}}+\frac{\boldsymbol{L}}{L^{2}}\left(R_{t} x-x\right) \cdot \boldsymbol{S} .
$$

The same expression can be written alternatively as

$$
\boldsymbol{x}(t)=\boldsymbol{x}+g t \boldsymbol{S} \times \boldsymbol{x}+\frac{\boldsymbol{L}}{L^{2}}\left(R_{t} \boldsymbol{x}-\boldsymbol{x}+g t \boldsymbol{L} \times \boldsymbol{x}\right) \cdot \boldsymbol{S} .
$$

In this form the connection with $\dot{\boldsymbol{x}}(0)=(\boldsymbol{x}, H)=g \boldsymbol{S} \times \boldsymbol{x}$ is more transparent since the last term is $O\left(t^{2}\right)$. Completely similar expressions apply for $\boldsymbol{k}(t)$.

Explicit solutions are also obtained for the special case in which $a$ and $\boldsymbol{b}$ are functions of $\boldsymbol{L}$ only, as this property is preserved during evolution with the spin-orbit Hamiltonian. This gives

$$
\begin{aligned}
& a(t)=a(0), \\
& \boldsymbol{b}(t)=\nabla a+R_{t}(\boldsymbol{b}(0)-\nabla a) .
\end{aligned}
$$

(The nablas refer a derivative with respect to $L$.) In this simpler setting we have explicitly checked that the canonical bracket is preserved during evolution, i.e., $(A(t), B(t))=(A, B)(t)$. 


\section{HYBRID LIE BRACKET}

In this section we show how hybrid brackets on $\mathscr{A}$, fulfilling the postulates of Eq. (2.24) and Eq. (2.9), can be constructed when the Hilbert space of the quantum sector is finite dimensional,

$$
\operatorname{dim} \mathscr{H}=n<\infty .
$$

In Appendix $\mathrm{A}$ we comment on slightly more general constructions.

\section{A. Definition of the bracket}

To construct the bracket, let us define $\tilde{\mathscr{A}}_{q} \subseteq \mathscr{A}_{q}$ as the set of traceless operators,

$$
\tilde{\mathscr{A}}_{q}=\left\{\tilde{Q} \in \mathscr{A}_{q}, \operatorname{tr}(\tilde{Q})=0\right\} .
$$

Then, any $Q \in \mathscr{A}_{q}$ can be uniquely decomposed as

$$
Q=Q_{c}+\tilde{Q}
$$

where $Q_{c} \propto \mathbb{1}$ and $\operatorname{tr}(\tilde{Q})=0$, with

$$
Q_{c} \equiv \frac{1}{n} \operatorname{tr}(Q) \mathbb{1} .
$$

In turn, the hybrid observables can be uniquely decomposed as

$$
A=C \mathbb{1}+\tilde{A}
$$

where $C$ is classical and $\tilde{A}$ is traceless. So

$$
\mathscr{A}=\mathscr{A}_{c} \oplus \tilde{\mathscr{A}},
$$

where $\tilde{\mathscr{A}}$ denotes the set of traceless hybrid operators. This set is $\tilde{\mathscr{A}}=\mathscr{A}_{c} \otimes \tilde{\mathscr{A}}_{q}$, i.e.,

$$
\tilde{A}=\sum_{i} C_{i} \tilde{Q}_{i}, \quad C_{i} \in \mathscr{A}_{c}, \quad \tilde{Q}_{i} \in \tilde{\mathscr{A}}_{q}
$$

The other property we need is that $\tilde{\mathscr{A}}_{q}$ is closed under commutation,

$$
\llbracket \tilde{\mathscr{A}}_{q}, \tilde{\mathscr{A}}_{q} \subseteq \tilde{\mathscr{A}}_{q} .
$$

This follows from $\operatorname{tr}\left(\left[Q_{1}, Q_{2}\right]\right)=0$ due to the cyclic property of the trace.

In these definitions it is easy to recognize the pattern of the example of the particle with classical position-momentum and quantum spin- $\frac{1}{2}$ considered in the previous section. There $\mathscr{A}_{c}$ is $\left\{A_{0}(\boldsymbol{x}, \boldsymbol{k}) \mathbb{1}\right\}$ and $\tilde{\mathscr{A}}$ is $\{\boldsymbol{A}(\boldsymbol{x}, \boldsymbol{k}) \cdot \boldsymbol{S}\}$.

Our statement is that a Lie bracket $($, ) can be defined in $\mathscr{A}$ as follows:

$$
\left(C+\tilde{A}, C^{\prime}+\tilde{A}^{\prime}\right):=\left\{C, C^{\prime}\right\}+\left\{\tilde{A}, C^{\prime}\right\}+\left\{C, \tilde{A}^{\prime}\right\}+\llbracket \tilde{A}, \tilde{A}^{\prime} \rrbracket .
$$

Here $\{$,$\} and \llbracket, \rrbracket$ are the usual classical and quantum dynamical brackets, and the $\mathbb{1}$ operator is implicit.

More explicitly

$$
\left(C \mathbb{1}+\sum_{i} C_{i} \tilde{Q}_{i}, C^{\prime} \mathbb{1}+\sum_{j} C_{j}^{\prime} \tilde{Q}_{j}^{\prime}\right)=\left\{C, C^{\prime}\right\} \mathbb{1}+\sum_{j}\left\{C, C_{j}^{\prime}\right\} \tilde{Q}_{j}^{\prime}+\sum_{i}\left\{C_{i}, C^{\prime}\right\} \tilde{Q}_{i}+\sum_{i, j} C_{i} C_{j}^{\prime} \llbracket \tilde{Q}_{i}, \tilde{Q}_{j}^{\prime} \rrbracket .
$$

Here, and also in the proof below, we use the symbols $\tilde{Q}$, $\tilde{Q}_{i}$, etc, to denote operators in $\tilde{\mathscr{A}}_{q}$ (rather than to arbitrary elements of $\mathscr{A}_{q}$ ). The symbols $C, C_{i}$, etc, will denote elements of $\mathscr{A}_{c}$, i.e., classical observables, these are c-number functions on the phase space.

The definition in Eq. (3.2) for a particle with classical position-momentum and quantum spin- $\frac{1}{2}$ is just an instance of the bracket in Eq. (4.10) for the general case. 


\section{B. Proof of the Lie bracket property}

Clearly, the construction in Eq. (4.9) is linear and antisymmetric and fulfills the axioms Eq. 2.24), as well as Eq. (2.9). We now show that it also fulfills the Jacobi identity. The Jacobi identity states that for any three hybrid observables $A_{1}, A_{2}$ and $A_{3}$,

$$
\left(A_{1},\left(A_{2}, A_{3}\right)\right)+\left(A_{2},\left(A_{3}, A_{1}\right)\right)+\left(A_{3},\left(A_{1}, A_{2}\right)\right)=0
$$

or equivalently (using antisymmetry)

$$
\left(A_{1},\left(A_{2}, A_{3}\right)\right)=\left(\left(A_{1}, A_{2}\right), A_{3}\right)+\left(A_{2},\left(A_{1}, A_{3}\right)\right) .
$$

For each observable it is sufficient to consider the two cases $A=C$ or $A=C \tilde{Q}$. The eight possibilities so generated can be classified by the number $n_{q}$ of $\tilde{Q}$ 's involved. It is only necessary to check the four cases $n_{q}=0,1,2,3$.

Case $n_{q}=0$ : For three classical observables $C_{1}, C_{2}$ and $C_{3}$ (as usual we omit the factor $\mathbf{1}$ )

$$
\left\{C_{1},\left\{C_{2}, C_{3}\right\}\right\}+\left\{C_{2},\left\{C_{3}, C_{1}\right\}\right\}+\left\{C_{3},\left\{C_{1}, C_{2}\right\}\right\}=0 .
$$

This is trivially true being $\{$,$\} a Lie bracket.$

Case $n_{q}=1$ : For $A_{1}=C_{1} \tilde{Q}_{1}$ and $A_{i}=C_{i}, i=2,3$,

$$
\begin{aligned}
\left(C_{1} \tilde{Q}_{1},\left(C_{2}, C_{3}\right)\right) & =\left\{C_{1},\left\{C_{2}, C_{3}\right\}\right\} \tilde{Q}_{1} \\
\left(\left(C_{1} \tilde{Q}_{1}, C_{2}\right), C_{3}\right)+\left(C_{2},\left(C_{1} \tilde{Q}_{1}, C_{3}\right)\right) & =\left(\left\{C_{1}, C_{2}\right\} \tilde{Q}_{1}, C_{3}\right)+\left(C_{2},\left\{C_{1}, C_{3}\right\} \tilde{Q}_{1}\right) \\
& =\left\{\left\{C_{1}, C_{2}\right\}, C_{3}\right\} \tilde{Q}_{1}+\left\{C_{2},\left\{C_{1}, C_{3}\right\}\right\} \tilde{Q}_{1} .
\end{aligned}
$$

The two expressions coincide since $\{$,$\} is a Lie bracket.$

Case $n_{q}=2$ : For $A_{1}=C_{1}$ and $A_{i}=C_{i} \tilde{Q}_{i}, i=2,3$,

$$
\left(C_{1},\left(C_{2} \tilde{Q}_{2}, C_{3} \tilde{Q}_{3}\right)\right)=\left(C_{1}, C_{2} C_{3} \llbracket \tilde{Q}_{2}, \tilde{Q}_{3} \rrbracket\right)=\left\{C_{1}, C_{2} C_{3}\right\} \llbracket \tilde{Q}_{2}, \tilde{Q}_{3} \rrbracket,
$$

using that $\llbracket \tilde{Q}_{2}, \tilde{Q}_{3} \rrbracket \in \tilde{\mathscr{A}}_{q}[\mathrm{Eq} .[4.8]$ in the second equality. On the other hand

$$
\begin{aligned}
\left(\left(C_{1}, C_{2} \tilde{Q}_{2}\right), C_{3} \tilde{Q}_{3}\right)+\left(C_{2} \tilde{Q}_{2},\left(C_{1}, C_{3} \tilde{Q}_{3}\right)\right) & =\left(\left\{C_{1}, C_{2}\right\} \tilde{Q}_{2}, C_{3} \tilde{Q}_{3}\right)+\left(C_{2} \tilde{Q}_{2},\left\{C_{1}, C_{3}\right\} \tilde{Q}_{3}\right) \\
& =\left\{C_{1}, C_{2}\right\} C_{3} \llbracket \tilde{Q}_{2}, \tilde{Q}_{3} \rrbracket+C_{2}\left\{C_{1}, C_{3}\right\} \llbracket \tilde{Q}_{2}, \tilde{Q}_{3} \rrbracket
\end{aligned}
$$

The expressions in (4.15) and (4.16) coincide since $\{$,$\} is a derivation and so \left\{C_{1}, C_{2} C_{3}\right\}=\left\{C_{1}, C_{2}\right\} C_{3}+C_{2}\left\{C_{1}, C_{3}\right\}$.

Case $n_{q}=3$ : For $A_{i}=C_{i} \tilde{Q}_{i}, i=1,2,3$,

$$
\left(C_{i} \tilde{Q}_{i},\left(C_{j} \tilde{Q}_{j}, C_{k} \tilde{Q}_{k}\right)\right)=\left(C_{i} \tilde{Q}_{i}, C_{j} C_{k} \llbracket \tilde{Q}_{j}, \tilde{Q}_{k} \rrbracket\right)=C_{i} C_{j} C_{k} \llbracket \tilde{Q}_{i}, \llbracket \tilde{Q}_{j}, \tilde{Q}_{k} \rrbracket \rrbracket .
$$

Clearly this expression vanishes upon adding cyclic permutations since this is true for $\llbracket$, \ and the $C$ 's commute.

This completes the proof.

\section{Observations}

The following observations are in order:

1) In the proof we have actually used only certain mathematical properties of $\mathscr{A}$ and $\tilde{\mathscr{A}}$. In Appendix A we comment on more general constructions relaxing the assumptions that $\mathscr{H}$ must be finite dimensional, $\mathscr{A}_{q}$ should contain all operators and $\tilde{\mathscr{A}}_{q}$ should be the subset of traceless operators in $\mathscr{A}_{q}$.

2) We have noted that there is no hybrid dynamical Lie bracket fulfilling the axioms in Eq. 2.24 when the classical and quantum sectors are of position-momentum type [25]. The impediment comes from a quantum sector with Hilbert space $\mathscr{H}=$ $L^{2}\left(\mathbb{R}^{n}\right)$. In this case the condition $\llbracket \tilde{\mathscr{A}}_{q}, \tilde{\mathscr{A}}_{q} \rrbracket \subseteq \tilde{\mathscr{A}}_{q}$ (used in the case $n_{q}=2$ ) is violated since the commutator of two operators produces in general a component along the $\mathbb{1}$. An obvious example is that of the canonical commutation relations $\llbracket q^{i}, p_{j} \rrbracket=\delta_{j}^{i} \mathbb{1}$.

3) The bracket in Eq. 4.9) is not a derivation. This can be seen by comparing our prescription

$$
\left(C \tilde{Q}, C^{\prime} \tilde{Q}^{\prime}\right)=C C^{\prime} \llbracket \tilde{Q}, \tilde{Q}^{\prime} \rrbracket .
$$

with Eq. 2.22, which has an extra term. 
4) The bracket in Eq. (4.9) is somewhat similar to the "standard" proposal $(,)_{s}$ in Eq. (2.14), but bypasses the problem of the Jacobi identity by treating separately the classical component $(C$ in $A=C+\tilde{A})$ and the quantum component $(\tilde{A})$ of the observables. With the "standard" bracket one would obtain the same result as with our Lie bracket for the cases covered by the axioms, i.e., when one of the observables is purely classical or purely quantal, while for the case of two properly hybrid observables

$$
\left(C \tilde{Q}, C^{\prime} \tilde{Q}^{\prime}\right)_{s}=C C^{\prime} \llbracket \tilde{Q}, \tilde{Q}^{\prime} \rrbracket+\frac{1}{2}\left(\tilde{Q} \tilde{Q}^{\prime}+\tilde{Q}^{\prime} \tilde{Q}\right)\left\{C, C^{\prime}\right\}
$$

which differs from our prescription in Eq. 4.18 by the last term.

5) It is easy to check that our bracket can also be expressed as

$$
\left(C Q, C^{\prime} Q^{\prime}\right)=C C^{\prime} \llbracket Q, Q^{\prime} \rrbracket+\left\{C, C^{\prime}\right\}\left(Q Q^{\prime}-\tilde{Q} \tilde{Q}^{\prime}\right),
$$

where $Q=Q_{c}+\tilde{Q}, Q^{\prime}=Q_{c}^{\prime}+\tilde{Q}^{\prime}$, with $Q_{c}, Q_{c}^{\prime} \propto \mathbb{1}$ and $\tilde{Q}, \tilde{Q}^{\prime} \in \tilde{A}_{q}$, and $C, C^{\prime} \in \mathscr{A}_{c}$. The factor $Q Q^{\prime}-\tilde{Q} \tilde{Q}^{\prime}$ is actually symmetric with respect to $Q \leftrightarrow Q^{\prime}$.

6) Our bracket can be written in form similar to that in Eq. 3.2), as follows. Let $\left\{q_{i}\right\}$, be a basis of $\tilde{\mathscr{A}}_{q}$ ( $\operatorname{similar}$ to $S_{i}=\hbar \sigma_{i} / 2$ for spin- $\frac{1}{2}$ ) then, because $\tilde{\mathscr{A}}_{q}$ defines a Lie algebra with the commutator,

$$
\llbracket q_{i}, q_{j} \rrbracket=c_{i j}{ }^{k} q_{k}
$$

for some structure constants. The elements of $\tilde{\mathscr{A}}_{q}$ can be written as $\boldsymbol{a}=\boldsymbol{a} \cdot \boldsymbol{q}=a^{i} q_{i}$ and so, using a standard notation,

$$
\llbracket a, b \rrbracket=\boldsymbol{a} \times \boldsymbol{b} \cdot \boldsymbol{q}, \quad(\boldsymbol{a} \times \boldsymbol{b})^{k} \equiv c_{i j}{ }^{k} a^{i} b^{j} .
$$

With this notation our hybrid bracket takes the form

$$
\left(A_{0}+\boldsymbol{A} \cdot \boldsymbol{q}, B_{0}+\boldsymbol{B} \cdot \boldsymbol{q}\right)=\left\{A_{0}, B_{0}\right\}+\left\{A_{0}, \boldsymbol{B}\right\} \cdot \boldsymbol{q}+\left\{\boldsymbol{A}, B_{0}\right\} \cdot \boldsymbol{q}+\boldsymbol{A} \times \boldsymbol{B} \cdot \boldsymbol{q} .
$$

7) It is interesting to note that for this bracket the product of a classical constant of motion $C$ with a generic hybrid observable $A$, gives

$$
(C A, H)=C(A, H) \quad \forall A \in \mathscr{A} \text {. }
$$

This follows from the fact that $\{$,$\} is a derivation and \left(\boldsymbol{C}, H_{0}\right)=(\boldsymbol{C}, \boldsymbol{H})=0$, where $H=H_{0}+\boldsymbol{H} \cdot \boldsymbol{q}$. An immediate consequence is that if $A$ is also a constant of motion, the product $C A$ is conserved too. Of course, similar assertions hold when $C$ is invariant under a transformation group.

\section{UNIQUENESS OF THE HYBRID BRACKET}

In this section we show that the hybrid bracket of Eq. 4.23) is the only solution to the double requirement of being a Lie bracket and fulfilling the axioms in Eq. 2.24). This rather technical section is entirely devoted to prove this.

We explicitly assume that the Hilbert space is finite-dimensional and $\mathscr{A}_{q}$ is the set of all operators. The uniqueness does not automatically hold in the generalizations discussed in Appendix A

Let $n=\operatorname{dim} \mathscr{H}$, so $\mathscr{A}_{q}$ is the set of $n \times n$ matrices. Any such matrix can be written as

$$
Q=a \mathbb{1}+\boldsymbol{b} \cdot \boldsymbol{\lambda}
$$

where the $\lambda_{i}, i=1, \ldots, n^{2}-1$ are the Gell-Mann matrices of $\operatorname{su}(n)$ (a generalization of the Pauli matrices) [53]. The Gell-Mann matrices are $n^{2}-1$ linearly independent Hermitian and traceless matrices which define a basis of $\tilde{\mathscr{A}}_{q}$ :

$$
\lambda_{i}=\lambda_{i}^{\dagger}, \quad \operatorname{tr}\left(\lambda_{i}\right)=0
$$

They fulfill the relations

$$
\lambda_{i} \lambda_{j}=\frac{2}{n} \delta_{i j}+d_{i j k} \lambda_{k}+i f_{i j k} \lambda_{k}
$$

where the tensor $d_{i j k}$ is fully symmetric and $f_{i j k}$ is fully antisymmetric. The $\lambda_{i}$ are normalized so that $f_{i l m} f_{j l m}=n \delta_{i j}$. 
The most general hybrid observable takes the form

$$
A=C+C \cdot \boldsymbol{q}
$$

where $C$ and $\boldsymbol{C}$ are c-number functions on the phase space and we have introduced the basis of $\tilde{\mathscr{A}}_{q}$

$$
q_{i} \equiv \frac{\hbar}{2} \lambda_{i}
$$

which fulfills the commutation relations

$$
\llbracket q_{i}, q_{j} \rrbracket=f_{i j k} q_{k}
$$

as follows from Eq. (5.3).

To fix the hybrid bracket we need to specify $\left(C, C^{\prime}\right),\left(C q_{i}, C^{\prime}\right)$ and $\left(C q_{i}, C^{\prime} q_{j}\right)$. (We already assume antisymmetry of the bracket.) The first two are immediate from the postulates,

$$
\left(C, C^{\prime}\right)=\left\{C, C^{\prime}\right\}, \quad\left(C q_{i}, C^{\prime}\right)=\left\{C, C^{\prime}\right\} q_{i},
$$

and the postulates also require

$$
\left(C q_{i}, q_{j}\right)=f_{i j k} C q_{k}
$$

The most general form of $\left(C q_{i}, C^{\prime} q_{j}\right)$ can be written as

$$
\left(C q_{i}, C^{\prime} q_{j}\right)=\left(C, C^{\prime}\right)_{i j} \mathbb{1}+\left(C, C^{\prime}\right)_{i j k} q_{k},
$$

where $\left(C, C^{\prime}\right)_{i j}$ and $\left(C, C^{\prime}\right)_{i j k}$ are two bilinear operations to be specified. To do this let us enforce the Jacobi identity for the three operators $C q_{i}, C^{\prime} q_{j}$ and $q_{k}$. On one hand

$$
\left(\left(C q_{i}, C^{\prime} q_{j}\right), q_{k}\right)=\left(C, C^{\prime}\right)_{i j l} f_{l k m} q_{m}
$$

while by Jacobi this equals

$$
\left(\left(C q_{i}, q_{k}\right), C^{\prime} q_{j}\right)+\left(C q_{i},\left(C^{\prime} q_{j}, q_{k}\right)\right)=f_{i k l}\left(C, C^{\prime}\right)_{l j}+f_{j k l}\left(C, C^{\prime}\right)_{i l}+f_{i k l}\left(C, C^{\prime}\right)_{l j m} q_{m}+f_{j k l}\left(C, C^{\prime}\right)_{i l m} q_{m} .
$$

This is equivalent to

$$
\begin{aligned}
& 0=f_{i k l}\left(C, C^{\prime}\right)_{l j}+f_{j k l}\left(C, C^{\prime}\right)_{i l}, \\
& 0=f_{i k l}\left(C, C^{\prime}\right)_{l j m}+f_{j k l}\left(C, C^{\prime}\right)_{i l m}+f_{m k l}\left(C, C^{\prime}\right)_{i j l} .
\end{aligned}
$$

The right-hand sides represent the infinitesimal $\mathrm{SU}(n)$ rotations of $\left(C, C^{\prime}\right)_{i j}$ and $\left(C, C^{\prime}\right)_{i j m}$, and so the equations imply that these are $\mathrm{SU}(n)$-invariant tensors. The only available invariant tensors of rank 2 and 3 are those in Eq. (5.3), thus this leads to

$$
\left(C q_{i}, C^{\prime} q_{j}\right)=\left(C, C^{\prime}\right)_{\delta} \delta_{i j} \mathbb{1}+\left(C, C^{\prime}\right)_{f} f_{i j k} q_{k}+\left(C, C^{\prime}\right)_{d} d_{i j k} q_{k} .
$$

Here $(,)_{\delta},(,)_{f}$ and $(,)_{d}$ are three auxiliary brackets to be specified. The antisymmetry of $($,$) requires$

$$
\begin{aligned}
& \left(C, C^{\prime}\right)_{\delta}=-\left(C^{\prime}, C\right)_{\delta} \\
& \left(C, C^{\prime}\right)_{f}=+\left(C^{\prime}, C\right)_{f} \\
& \left(C, C^{\prime}\right)_{d}=-\left(C^{\prime}, C\right)_{d}
\end{aligned}
$$

Further, Eq. (5.8) requires

$$
(C, 1)_{\delta}=(C, 1)_{d}=0, \quad(C, 1)_{f}=C .
$$

Of course, our hybrid bracket in Eq. (4.23) fulfills all these conditions. Specifically, for our hybrid bracket

$$
\left(C, C^{\prime}\right)_{\delta}=\left(C, C^{\prime}\right)_{d}=0, \quad\left(C, C^{\prime}\right)_{f}=C C^{\prime} .
$$

We want to show that this is actually the unique solution for a Lie bracket fulfilling the constraints in Eqs. (5.14) and (5.15). 
To restrict the form of the auxiliary brackets $(,)_{\delta},(,)_{f}$, and $(,)_{d}$, we need to fully enforce the Jacobi identity for three generic hybrid operators. It is easy to verify that if there are zero or one $q_{i}$ involved, Jacobi is fulfilled automatically. So the first non trivial constraint comes from three operators with two $q_{i}$ :

$$
\left(\left(C q_{i}, C^{\prime} q_{j}\right), C^{\prime \prime}\right)=\left(\left(C q_{i}, C^{\prime \prime}\right), C^{\prime} q_{j}\right)+\left(C q_{i},\left(C^{\prime} q_{j}, C^{\prime \prime}\right)\right) .
$$

Expanding this expression using Eqs. (5.7) and (5.13), and noting that the three tensors $\delta_{i j}, f_{i j k}$ and $d_{i j k}$ are linearly independent, leads to the relations

$$
\begin{aligned}
& \left\{\left(C, C^{\prime}\right)_{\delta}, C^{\prime \prime}\right\}=\left(\left\{C, C^{\prime \prime}\right\}, C^{\prime}\right)_{\delta}+\left(C,\left\{C^{\prime}, C^{\prime \prime}\right\}\right)_{\delta}, \\
& \left\{\left(C, C^{\prime}\right)_{f}, C^{\prime \prime}\right\}=\left(\left\{C, C^{\prime \prime}\right\}, C^{\prime}\right)_{f}+\left(C,\left\{C^{\prime}, C^{\prime \prime}\right\}\right)_{f}, \\
& \left\{\left(C, C^{\prime}\right)_{d}, C^{\prime \prime}\right\}=\left(\left\{C, C^{\prime \prime}\right\}, C^{\prime}\right)_{d}+\left(C,\left\{C^{\prime}, C^{\prime \prime}\right\}\right)_{d} .
\end{aligned}
$$

In other words, the Poisson bracket acts as a derivation (in the sense of Lie products [54]) with respect to the auxiliary brackets. In Appendix B we show that these conditions have as unique solutions [using the symmetry conditions in Eq. $[5.14 \text { ] }]^{12}$

$$
\left(C, C^{\prime}\right)_{\delta}=\alpha\left\{C, C^{\prime}\right\}, \quad\left(C, C^{\prime}\right)_{f}=\beta C C^{\prime}, \quad\left(C, C^{\prime}\right)_{d}=\gamma\left\{C, C^{\prime}\right\},
$$

for some arbitrary constants $\alpha, \beta$ and $\gamma$. (Our bracket corresponds to $\alpha=\gamma=0$ and $\beta=1$.)

The value $\beta=1$ follows from Eq. (5.15). To fix $\alpha$ and $\gamma$ we proceed to impose Jacobi with three $q_{i}$. Using previous results one obtains (for convenience we retain an arbitrary $\beta$ )

$$
\begin{aligned}
\left(C q_{i},\left(C^{\prime} q_{j}, C^{\prime \prime} q_{k}\right)\right)= & \alpha \beta\left\{C, C^{\prime} C^{\prime \prime}\right\} f_{i j k}+\alpha \gamma\left\{C,\left\{C^{\prime}, C^{\prime \prime}\right\}\right\} d_{i j k}+\alpha\left\{C,\left\{C^{\prime}, C^{\prime \prime}\right\}\right\} \delta_{j k} \delta_{i m} q_{m} \\
& +\beta^{2} C C^{\prime} C^{\prime \prime} f_{j k l} f_{i l m} q_{m}+\beta \gamma\left\{C, C^{\prime} C^{\prime \prime}\right\} f_{j k l} d_{i l m} q_{m}+\beta \gamma C\left\{C^{\prime}, C^{\prime \prime}\right\} d_{j k l} f_{i l m} q_{m}+\gamma^{2}\left\{C, C^{\prime} C^{\prime \prime}\right\} d_{j k l} d_{i l m} q_{m} .
\end{aligned}
$$

This expression must vanish upon summation on its three cyclic permutations. Doing this, the terms with $\alpha \beta, \alpha \gamma$ and $\beta^{2}$ cancel identically. This leaves, for all $C, C^{\prime}$ and $C^{\prime \prime}$, and all $i, j, k, m$,

$$
0=\alpha\left\{C,\left\{C^{\prime}, C^{\prime \prime}\right\}\right\} \delta_{j k} \delta_{i m}+\beta \gamma\left\{C, C^{\prime} C^{\prime \prime}\right\} f_{j k l} d_{i l m}+\beta \gamma C\left\{C^{\prime}, C^{\prime \prime}\right\} d_{j k l} f_{i l m}+\gamma^{2}\left\{C, C^{\prime} C^{\prime \prime}\right\} d_{j k l} d_{i l m}+\text { c.p. },
$$

where c.p. stands for cyclic permutations of $\left(\{C, i\},\left\{C^{\prime}, j\right\},\left\{C^{\prime \prime}, k\right\}\right)$.

For $n \geq 4$ the rank-4 invariant tensors span a nine-dimensional space, ${ }^{13}$ and a basis is given by [53]

$$
\begin{array}{lll}
\delta_{j k} \delta_{i m}, & \delta_{k i} \delta_{j m}, & \delta_{i j} \delta_{k m}, \\
d_{j k l} d_{i l m}, & d_{k i l} d_{j l m}, & d_{i j l} d_{k l m}, \\
d_{j k l} f_{i l m}, & d_{k i l} f_{j l m}, & f_{i j l} d_{k l m} .
\end{array}
$$

As a consequence, the vanishing of the components along $\delta_{j k} \delta_{i m}$ and $d_{j k l} d_{i l m}$ in Eq. 5.21) requires

$$
0=\alpha\left\{C,\left\{C^{\prime}, C^{\prime \prime}\right\}\right\}, \quad 0=\gamma^{2}\left\{C, C^{\prime} C^{\prime \prime}\right\} .
$$

Since $\left\{C,\left\{C^{\prime}, C^{\prime \prime}\right\}\right\}$ or $\left\{C, C^{\prime} C^{\prime \prime}\right\}$ need not be zero, it follows that $\alpha=\gamma=0$, which is our bracket (together with $\beta=1$ ). For these values all terms in Eq. (5.21) vanish as well, and this checks again that ours is a Lie bracket.

When $n=3$ the space of rank-4 invariant tensors span an eight-dimensional space, due to the relation

$$
\delta_{j k} \delta_{i m}+\delta_{k i} \delta_{j m}+\delta_{i j} \delta_{k m}=3\left(d_{j k l} d_{i l m}+d_{k i l} d_{j l m}+d_{i j l} d_{k l m}\right) .
$$

Eliminating $\delta_{i j} \delta_{k m}$, the vanishing of the components along $\delta_{j k} \delta_{i m}$ and $d_{j k l} d_{i l m}$ in Eq. (5.21) implies

$$
0=\alpha\left(\left\{C,\left\{C^{\prime}, C^{\prime \prime}\right\}\right\}-\left\{C^{\prime \prime},\left\{C, C^{\prime}\right\}\right\}\right), \quad 0=\gamma^{2}\left\{C, C^{\prime} C^{\prime \prime}\right\}-3 \alpha\left\{C,\left\{C^{\prime}, C^{\prime \prime}\right\}\right\} .
$$

Once again the brackets need not vanish ${ }^{14}$ so $\alpha=\gamma=0$.

\footnotetext{
${ }^{12}$ In the proof of Appendix B we explicitly assume that the classical sector is of position-momentum type. It is not obvious whether the same proof covers more general cases.

${ }^{13}$ This is just the number of times that the singlet representation appears in the direct product of four adjoint representations of $\mathrm{SU}(n)$.

${ }^{14}$ For instance, for $C=x^{2}, C^{\prime}=k^{2}$ and $C^{\prime \prime}=x k,\left\{C,\left\{C^{\prime}, C^{\prime \prime}\right\}\right\}-\left\{C^{\prime \prime},\left\{C, C^{\prime}\right\}\right\}=-8 x k$.
} 
For $n=2, d_{i j k}=0\left((,)_{d}\right.$ does not appear $)$ and the space has dimension three with $\delta_{j k} \delta_{i m}, \delta_{k i} \delta_{j m}$, and $\delta_{i j} \delta_{k m}$ as basis. Eq. 5.21) requires $\alpha=0$.

Throughout we have implicitly assumed $n>1$. When $\operatorname{dim} \mathscr{H}=1$ the $q_{i}$ do not exist. There is just one quantum state which is therefore completely invariant under evolution or transformations. The dynamical bracket reduces to the classical one.

This completes the proof of uniqueness.

It is interesting that a proof along the same lines can be provided for a quantal-quantal bracket. This means the following: Let us have two quantum systems provided with their usual dynamical bracket (the commutator), and let us try to assign the most general dynamical bracket to the compound system restricted by postulates similar to those imposed in the quantum-classical hybrid case. Then one finds that the commutator is the unique solution as a consistent dynamical bracket of the compound system. We have checked this when one of the sectors is of position-momentum type and the other a finite-dimensional Hilbert space (adapting the proof given in this section). As shown in [25] the same holds when the two quantum sectors are of positionmomentum type and also in a classical-classical system, i.e., only the Poisson bracket is consistent as dynamical bracket of the classical compound system.

\section{THE SCHRODINGER PICTURE}

\section{A. Definition of the hybrid bracket in the Schrodinger picture}

Up to now we have been studying the evolution of hybrid systems within the Heisenberg picture, in this section we obtain the dual description in the Schrodinger picture, in which the density matrix $\rho$ carries the dynamical evolution. To this end let us introduce the notation

$$
\langle A\rangle\rangle \equiv \int d^{n} x d^{n} k \operatorname{tr}(A(x, k)),
$$

that is, the symbol $\langle\langle\rangle$ denotes integration over the classical phase space plus trace over the quantum Hilbert space. In this way Eq. (2.6) can be rewritten as

$$
\langle A\rangle_{\rho}=\langle\langle A \rho\rangle,
$$

where $\rho(x, k ; t)$ is the density matrix of the hybrid system.

Throughout this section we assume that the Hilbert space is finite dimensional, and as in the rest of the paper (except Appendix [A], $\mathscr{A}_{q}$ contains all operators and $\tilde{A}_{q}$ is the subset of traceless operators. In this case

$$
\langle\tilde{A}\rangle=0 \quad \forall \tilde{A} \in \tilde{\mathscr{A}} .
$$

Further properties are

$$
\langle\langle A B\rangle=\langle\langle B A\rangle,
$$

and also

$$
\langle\langle\{A, B\}\rangle=\langle\langle\llbracket A, B \rrbracket\rangle=0,
$$

using integration by parts in the first case and the cyclic property of the trace in the second case. More generally,

$$
\left\langle\left\langle\left\{A_{1}, A_{2}\right\} A_{3}\right\rangle\right\rangle=\left\langle\left\langle A_{1}\left\{A_{2}, A_{3}\right\}\right\rangle, \quad\left\langle\left\langle\llbracket A_{1}, A_{2} \rrbracket A_{3}\right\rangle\right\rangle=\left\langle\left\langle A_{1} \llbracket A_{2}, A_{3} \rrbracket\right\rangle .\right.\right.
$$

In order to obtain the evolution of $\rho$, we postulate as usual that the expectation value must coincide in both pictures. Therefore

$$
\frac{d}{d t}\langle A\rangle_{\rho}=\left\langle\left\langle\frac{d A}{d t} \rho\right\rangle\right\rangle=\left\langle\left\langle A \frac{d \rho}{d t}\right\rangle,\right.
$$

in the Heisenberg and Schrodinger pictures, respectively. As a consequence

$$
\left\langle\left\langle A \frac{d \rho}{d t}\right\rangle=\langle\langle(A, H) \rho\rangle .\right.
$$

This holds for any $A$, and so $\rho$ obeys a certain linear equation which can be written in the form

$$
\frac{d \rho}{d t}=(H, \rho)^{\prime}
$$


Here $(A, B)^{\prime}$ is an operation which is linear in $A$ and $B$. The symbol $(,)^{\prime}$ is the dynamical bracket in the Schrodinger picture. From the previous relations, it follows that this bracket satisfies the condition

$$
\left\langle\left\langle A(H, \rho)^{\prime}\right\rangle\right\rangle=\langle\langle(A, H) \rho\rangle .
$$

Since $A$ is completely arbitrary, this relation fully fixes $(H, \rho)^{\prime}$ : indeed, $\langle\langle A B\rangle\rangle=0$ for all $A$ can only hold if $B=0$, and so $\left\langle\left\langle A B_{1}\right\rangle\right\rangle=\left\langle\left\langle A B_{2}\right\rangle\right\rangle$ for all $A$ implies $B_{1}=B_{2}$. Alternatively,

$$
\frac{\delta\langle\langle A B\rangle\rangle}{\delta A}=B
$$

implies that $B$ is fixed from the knowledge of $\langle\langle A B\rangle$ for all $A$.

Let us note that when all observables are classical $\langle\langle\{A, H\} \rho\rangle=\langle\langle A\{H, \rho\}\rangle[$ using Eq. 6.6 ]. Likewise in the quantum case $\langle\langle\llbracket A, H \rrbracket \rho\rangle=\langle\langle A \llbracket H, \rho \rrbracket\rangle$. So in these two cases the bracket takes the same form in the Heisenberg and Schrodinger pictures:

$$
\{,\}^{\prime}=\{,\}, \quad \llbracket, \rrbracket^{\prime}=\llbracket, \rrbracket .
$$

However, in the hybrid case $(,)^{\prime}$ needs not coincide with $($,$) , and in fact it does not.$

\section{B. Explicit construction of the hybrid bracket in the Schrodinger picture}

In order to fully determine the bracket from Eq. 6.10), we will consider the most general hybrid operator $A$, which is of the type $C+C^{\prime} \tilde{Q}$, with $C, C^{\prime} \in \mathscr{A}_{c}$ and $\tilde{Q} \in \tilde{\mathscr{A}}_{q}$ (that is, $\operatorname{tr}(\tilde{Q})=0$ ). We follow a procedure similar to that in the proof of the Jacobi identity, namely, we workout the various cases classified by the number $n_{q}$ of $\tilde{Q}$ 's present in the operators $\rho$ and $H$ in Eq. 6.10).

Case $n_{q}=0$ : For arbitrary $C_{i}$ and $\tilde{Q}_{i}$

$$
\begin{gathered}
\left\langle\left\langle C_{1}\left(C_{2}, C_{3}\right)^{\prime}\right\rangle\right\rangle=\left\langle\left\langle\left(C_{1}, C_{2}\right) C_{3}\right\rangle\right\rangle=\left\langle\left\langle C_{1}\left\{C_{2}, C_{3}\right\}\right\rangle,\right. \\
\left\langle\left\langle C_{1} \tilde{Q}_{1}\left(C_{2}, C_{3}\right)^{\prime}\right\rangle\right\rangle=\left\langle\left\langle\left(C_{1} \tilde{Q}_{1}, C_{2}\right) C_{3}\right\rangle\right\rangle=\left\langle\left\langle C_{1} \tilde{Q}_{1}\left\{C_{2}, C_{3}\right\}\right\rangle,,\right.
\end{gathered}
$$

where we have used Eq. (6.6). Hence, for all $A\left\langle\left\langle A\left(C_{2}, C_{3}\right)^{\prime}\right\rangle\right\rangle=\left\langle\left\langle A\left\{C_{2}, C_{3}\right\}\right\rangle\right\rangle$ and so

$$
\left(C_{2}, C_{3}\right)^{\prime}=\left\{C_{2}, C_{3}\right\}
$$

Case $n_{q}=1$. Since we do not assume that $(,)^{\prime}$ is antisymmetric, we have to distinguish two cases, depending on whether the $\tilde{Q}$ is in $H$ or in $\rho$. In the first instance

$$
\begin{gathered}
\left\langle\left\langle C_{1}\left(C_{2} \tilde{Q}_{2}, C_{3}\right)^{\prime}\right\rangle\right\rangle=\left\langle\left\langle\left(C_{1}, C_{2} \tilde{Q}_{2}\right) C_{3}\right\rangle\right\rangle=\left\langle\left\langle C_{1}\left\{C_{2}, C_{3}\right\} \tilde{Q}_{2}\right\rangle\right\rangle=0, \\
\left\langle\left\langle C_{1} \tilde{Q}_{1}\left(C_{2} \tilde{Q}_{2}, C_{3}\right)^{\prime}\right\rangle\right\rangle=\left\langle\left\langle\left(C_{1} \tilde{Q}_{1}, C_{2} \tilde{Q}_{2}\right) C_{3}\right\rangle\right\rangle=\left\langle\left\langle C_{1} C_{2} C_{3} \llbracket \tilde{Q}_{1}, \tilde{Q}_{2} \rrbracket\right\rangle=0 .\right.
\end{gathered}
$$

Where it has been used that $\operatorname{tr}(\tilde{Q})=\operatorname{tr}([A, B])=0$. Therefore

$$
\left(C_{2} \tilde{Q}_{2}, C_{3}\right)^{\prime}=0
$$

On the other hand, when the factor $\tilde{Q}$ is in $\rho$

$$
\begin{aligned}
\left\langle\left\langle C_{1}\left(C_{2}, C_{3} \tilde{Q}_{3}\right)^{\prime}\right\rangle\right\rangle & =\left\langle\left\langle\left(C_{1}, C_{2}\right) C_{3} \tilde{Q}_{3}\right\rangle\right\rangle=\left\langle\left\langle C_{1}\left\{C_{2}, C_{3}\right\} \tilde{Q}_{3}\right\rangle\right\rangle \\
\left\langle\left\langle C_{1} \tilde{Q}_{1}\left(C_{2}, C_{3} \tilde{Q}_{3}\right)^{\prime}\right\rangle\right\rangle & =\left\langle\left\langle\left(C_{1} \tilde{Q}_{1}, C_{2}\right) C_{3} \tilde{Q}_{3}\right\rangle\right\rangle=\left\langle\left\langle C_{1} \tilde{Q}_{1}\left\{C_{2}, C_{3}\right\} \tilde{Q}_{3}\right\rangle\right\rangle .
\end{aligned}
$$

Hence,

$$
\left(C_{2}, C_{3} \tilde{Q}_{3}\right)^{\prime}=\left\{C_{2}, C_{3}\right\} \tilde{Q}_{3} .
$$

Case $n_{q}=2$. In this case we derive the following relations

$$
\begin{aligned}
\left\langle C_{1}\left(C_{2} \tilde{Q}_{2}, C_{3} \tilde{Q}_{3}\right)^{\prime}\right\rangle & \left.=\left\langle\left(C_{1}, C_{2} \tilde{Q}_{2}\right) C_{3} \tilde{Q}_{3}\right\rangle\right\rangle=\left\langle\left\langle C_{1}\left\{C_{2}, C_{3}\right\} \tilde{Q}_{2} \tilde{Q}_{3}\right\rangle\right\rangle, \\
\left.\left\langle C_{1} \tilde{Q}_{1}\left(C_{2} \tilde{Q}_{2}, C_{3} \tilde{Q}_{3}\right)^{\prime}\right\rangle\right\rangle & \left.=\left\langle\left(C_{1} \tilde{Q}_{1}, C_{2} \tilde{Q}_{2}\right) C_{3} \tilde{Q}_{3}\right\rangle\right\rangle=\left\langle\left\langle C_{1} \tilde{Q}_{1} C_{2} C_{3} \llbracket \tilde{Q}_{2}, \tilde{Q}_{3} \rrbracket\right\rangle .\right.
\end{aligned}
$$

The second relation implies

$$
\left(C_{2} \tilde{Q}_{2}, C_{3} \tilde{Q}_{3}\right)^{\prime}=C_{2} C_{3} \llbracket \tilde{Q}_{2}, \tilde{Q}_{3} \rrbracket+C
$$


where $C$ is some element of $\mathscr{A}_{c}$. This ambiguity follows from $\left\langle\left\langle C_{1} \tilde{Q}_{1} C\right\rangle=0\right.$. On the other hand, from the first relation it follows that

$$
\left(C_{2} \tilde{Q}_{2}, C_{3} \tilde{Q}_{3}\right)^{\prime}=\left\{C_{2}, C_{3}\right\} \tilde{Q}_{2} \tilde{Q}_{3}+\tilde{A}
$$

where $\tilde{A}$ is some undetermined element of $\tilde{\mathscr{A}}$, since $\left\langle\left\langle C_{1} \tilde{A}\right\rangle\right\rangle=0$. To fulfill both conditions we rewrite this relation in the form

$$
\left(C_{2} \tilde{Q}_{2}, C_{3} \tilde{Q}_{3}\right)^{\prime}=\left\{C_{2}, C_{3}\right\} \frac{1}{n} \operatorname{tr}\left(\tilde{Q}_{2} \tilde{Q}_{3}\right)+\tilde{A}^{\prime}
$$

using that $\tilde{Q}_{2} \tilde{Q}_{3}=\frac{1}{n} \operatorname{tr}\left(\tilde{Q}_{2} \tilde{Q}_{3}\right)+\tilde{Q}^{\prime}$, with $\tilde{Q}^{\prime}$ traceless. Here $n=\operatorname{dim} \mathscr{H}=\operatorname{tr}(\mathbb{1})$. The conditions in Eqs. 6.20) and 6.22) are consistent identifying $C$ with $\left\{C_{2}, C_{3}\right\} \frac{1}{n} \operatorname{tr}\left(\tilde{Q}_{2} \tilde{Q}_{3}\right)$ and $\tilde{A}^{\prime}$ with $C_{2} C_{3} \llbracket \tilde{Q}_{2}, \tilde{Q}_{3} \rrbracket$, so finally

$$
\left(C_{2} \tilde{Q}_{2}, C_{3} \tilde{Q}_{3}\right)^{\prime}=C_{2} C_{3} \llbracket \tilde{Q}_{2}, \tilde{Q}_{3} \rrbracket+\left\{C_{2}, C_{3}\right\} \frac{1}{n} \operatorname{tr}\left(\tilde{Q}_{2} \tilde{Q}_{3}\right)
$$

In summary, the dynamical bracket in the Schrodinger picture takes the form

$$
\left(C_{1}+C_{1}^{\prime} \tilde{Q}_{1}, C_{2}+C_{2}^{\prime} \tilde{Q}_{2}\right)^{\prime}=\left\{C_{1}, C_{2}\right\}+\left\{C_{1}, C_{2}^{\prime}\right\} \tilde{Q}_{2}+C_{1}^{\prime} C_{2}^{\prime} \llbracket \tilde{Q}_{1}, \tilde{Q}_{2} \rrbracket+\left\{C_{1}^{\prime}, C_{2}^{\prime}\right\} \frac{1}{n} \operatorname{tr}\left(\tilde{Q}_{1} \tilde{Q}_{2}\right) .
$$

This reduces to the classical or quantum brackets when the observables are purely classical or purely quantal, respectively, as it is easily verified.

The density matrix must fulfill the conditions

$$
\left\langle\langle\rho\rangle=1, \quad \rho^{\dagger}=\rho .\right.
$$

These relations are preserved by the evolution induced by the bracket since

$$
\left\langle\left\langle(H, \rho)^{\prime}\right\rangle\right\rangle \equiv 0
$$

using Eq. 6.5, and also

$$
\left(A^{\dagger}, B^{\dagger}\right)^{\prime}=(A, B)^{\prime \dagger} \text {. }
$$

The dynamical bracket in the Schrodinger picture is not a Lie bracket (is not even antisymmetric). Also $(H,)^{\prime}$ is not a derivation. For instance, for a derivation one would have (with $H=C_{1}^{\prime} \tilde{Q}_{1}$ )

$$
\left(C_{1}^{\prime} \tilde{Q}_{1}, C_{2}^{\prime} \tilde{Q}_{2}\right)^{\prime}=\left(C_{1}^{\prime} \tilde{Q}_{1}, C_{2}^{\prime}\right)^{\prime} \tilde{Q}_{2}+C_{2}^{\prime}\left(C_{1}^{\prime} \tilde{Q}_{1}, \tilde{Q}_{2}\right)^{\prime}=0+C_{2}^{\prime} C_{1}^{\prime} \llbracket \tilde{Q}_{1}, \tilde{Q}_{2} \rrbracket
$$

while actually

$$
\left(C_{1}^{\prime} \tilde{Q}_{1}, C_{2}^{\prime} \tilde{Q}_{2}\right)^{\prime}=\left\{C_{1}^{\prime}, C_{2}^{\prime}\right\} \frac{1}{n} \operatorname{tr}\left(\tilde{Q}_{1} \tilde{Q}_{2}\right)+C_{2}^{\prime} C_{1}^{\prime} \llbracket \tilde{Q}_{1}, \tilde{Q}_{2} \rrbracket .
$$

The Schrodinger picture hybrid bracket can be written in a form similar to Eq. (4.23), as

$$
\left(A_{0}+\boldsymbol{A} \cdot \boldsymbol{q}, B_{0}+\boldsymbol{B} \cdot \boldsymbol{q}\right)^{\prime}=\left\{A_{0}, B_{0}\right\}+\left\{A_{0}, \boldsymbol{B}\right\} \cdot \boldsymbol{q}+\boldsymbol{A} \times \boldsymbol{B} \cdot \boldsymbol{q}+\frac{\hbar^{2}}{2 n}\{\boldsymbol{A} \cdot \boldsymbol{B}\},
$$

where, for $a=a^{i} q_{i}$ and $b=b^{i} q_{i}$

$$
\boldsymbol{a} \cdot \boldsymbol{b} \equiv g_{i j} a^{i} b^{j}, \quad g_{i j}=\frac{2}{\hbar^{2}} \operatorname{tr}\left(q_{i} q_{j}\right)
$$

\section{Classical particle with classical position-momentum and quantum spin- $\frac{1}{2}$}

For illustration purposes, let us workout the evolution of $\rho(t)$ for the Hamiltonian $H=g \boldsymbol{L} \cdot \boldsymbol{S}$, discussed in Sec. III. The general form of the density matrix is

$$
\rho(\boldsymbol{x}, \boldsymbol{k} ; t)=\alpha(\boldsymbol{x}, \boldsymbol{k} ; t)+\boldsymbol{\beta}(\boldsymbol{x}, \boldsymbol{k} ; t) \cdot \boldsymbol{S} .
$$


The equation of motion 6.9 leads to

$$
\frac{\partial \alpha}{\partial t}=\frac{\hbar^{2}}{4} g\{\boldsymbol{L} \cdot \boldsymbol{\beta}\}, \quad \frac{\partial \boldsymbol{\beta}}{\partial t}=g \boldsymbol{L} \times \boldsymbol{\beta} .
$$

These equations can be solved to give

$$
\begin{aligned}
& \alpha(t)=\alpha(0)-\frac{\hbar^{2}}{4 L^{2}} \operatorname{tr}\left(R_{t}^{-1}-1\right) \boldsymbol{L} \cdot \boldsymbol{\beta}(0)+\frac{\hbar^{2}}{4 L^{2}}\left(g t L_{i} L_{j}-\left(R_{t}^{-1}-1\right)_{i l} \varepsilon_{l m j} L_{m}\right) h_{i j}, \\
& \boldsymbol{\beta}(t)=R_{t}^{-1} \boldsymbol{\beta}(0),
\end{aligned}
$$

where $R_{t}$ is the same rotation as in Sec. III and

$$
h_{i j} \equiv\left\{L_{i}, \beta_{j}(0)\right\} .
$$

The expectation value with an observable $A=a+b \cdot S$ takes the form

$$
\left\langle\langle A \rho\rangle=\left\langle\left\langle a \alpha+\frac{\hbar^{2}}{4} b \cdot \boldsymbol{\beta}\right\rangle .\right.\right.
$$

The equivalence of the Heisenberg and Schrodinger pictures requires

$$
\left\langle\left\langle a(t) \alpha(0)+\frac{\hbar^{2}}{4} \boldsymbol{b}(t) \cdot \boldsymbol{\beta}(0)\right\rangle=\left\langle\left\langle a(0) \alpha(t)+\frac{\hbar^{2}}{4} \boldsymbol{b}(0) \cdot \boldsymbol{\beta}(t)\right\rangle\right\rangle .\right.
$$

Using integration by parts [Eq. [6.6] one can verify, after some algebra, that Eqs. 3.17) and 6.34 fulfill these relations.

In the simpler setting of $\rho$ being a function of $L$ only (a property preserved by the evolution with the spin-orbit dynamics) one obtains

$$
\begin{aligned}
& \alpha(t)=\alpha(0)+\frac{\hbar^{2}}{4} \nabla \cdot\left(R_{t}^{-1}-1\right) \boldsymbol{\beta}(0), \\
& \boldsymbol{\beta}(t)=R_{t}^{-1} \boldsymbol{\beta}(0) .
\end{aligned}
$$

From Eq. (6.36) and the formula of $\alpha(t)$ it is readily verified that $\left\langle\boldsymbol{L}^{2}\right\rangle_{\rho(t)}=\left\langle\left\langle\boldsymbol{L}^{2} \alpha(t)\right\rangle\right\rangle$ is independent of $t$ for any initial $\rho(0)$.

\section{THE POSITIVITY FIASCO}

As we have just shown, rather general requirements seem to completely fix in a unique way the form of the hybrid dynamics. In this section we show that unfortunately the requirement of positivity is not fulfilled by such dynamics. The same problem arises in the standard bracket of Eq. 2.14] [23]. Similar problems with positivity have been discussed in [55].

\section{A. Heisenberg picture}

In the Heisenberg picture, the requirement of positivity is a particular case of the more general requirement of the preservation of the range of an observable.

In quantum dynamics the evolution of an observable takes the form

$$
A(t)=U(t)^{-1} A U(t)
$$

where $U(t)$ denotes the evolution operator from time zero to time $t$. Since this is a similarity transformation, the spectrum of the observable is unchanged during the evolution, so the range (in the sense of spectrum) is preserved.

In the classical case, the range of an observable $A$ is the range of the function $A(x, k)$, i.e., the set of values that this quantity can take when $(x, k)$ is varied. This range is preserved by the classical evolution. Indeed, the evolved observable at time $t$, $A(x, k ; t)$, is nothing else than $A(x(t), k(t))$, where $x(t)$ and $k(t)$ are the evolutions of $x$ and $k$ (i.e., the solution of the Hamilton equations). Since that evolution is a bijection in the phase space the range of $A$ is unchanged. For the discussion of the hybrid case below, it is interesting to see the same thing from another point of view. Let us assume for simplicity that the range at time $t$ is $\left[a, \infty\left[\right.\right.$ and $A(t)$ attains the minimum value $a$ at $\left(x_{0}, k_{0}\right)$. In order for the range to change at $a$ one must have $\partial_{t} A\left(x_{0}, k_{0} ; t\right)$ different from zero, however the classical equation is

$$
\partial_{t} A(x, k ; t)=\nabla_{x} A(x, k ; t) \cdot \nabla_{k} H(x, k ; t)-\nabla_{k} A(x, k ; t) \cdot \nabla_{x} H(x, k ; t) .
$$


So $\nabla_{x} A$ and $\nabla_{k} A$ vanish at the minimum $\left(x_{0}, k_{0}\right)$ and so does $\partial_{t} A(x, k ; t)$.

The reason for the preservation of the range, either in the classical or the quantum cases, is clear. The range of the observable is the set of all possible values it can take in any of the states of the system. The evolution corresponds to a change of the state of the system, but a change of state cannot modify the range since it already accounts for all possible values. In this view it seems natural to demand the preservation of the range of observables during evolution also in the hybrid case.

For a hybrid observable we define its range as the union of all quantum spectra from all points of the classical phase space. Another equivalent definition of the range of $A$ is the set of values $\langle A\rangle_{\rho}$ can take as $\rho$ varies (restricted by the conditions of positivity and normalization). This range must not change during the evolution. In particular, if $A$ is positive (as an operator at all points $(x, k))$ this property ought to be preserved. However, this is not true for our dynamical hybrid bracket.

A simple counterexample (of preservation of positivity) follows from the particle with classical position-momentum and quantum spin- $\frac{1}{2}$ considered in Sec. III] Let us consider a generic observable $A=a+b \cdot \boldsymbol{\sigma}$ [Eq. (3.13)]. Such observable will be positive at $t=0$ provided its spectrum is positive at all $(\boldsymbol{x}, \boldsymbol{k})$. Since the local spectrum is $a \pm\|\boldsymbol{b}\|$, positivity requires

$$
a(\boldsymbol{x}, \boldsymbol{k} ; 0) \geq\|\boldsymbol{b}(\boldsymbol{x}, \boldsymbol{k} ; 0)\| \quad \forall \boldsymbol{x}, \boldsymbol{k} .
$$

For the dynamics $H=g \boldsymbol{L} \cdot \boldsymbol{S}$, the evolution is indicated in Eq. (3.17). There we can see in the equation for $\boldsymbol{b}(t)$, that the first and third terms are bounded components as they depend on $R_{t}$, however, the second term depends linearly with $t$. This term, which needs not vanish, will eventually dominate for large (positive or negative) $t$ and will spoil the positivity condition $a(t) \geq\|\boldsymbol{b}(t)\|$. This shows that positivity is not preserved in general by the hybrid dynamics.

The previous argument would not directly apply to a dynamics like that in Eq. 3.21) (with phase space based on $\boldsymbol{L}$ only), nevertheless, also in this case one can find instances of observables which are positive at $t=0$ but not at all later times.

\section{B. Schrodinger picture}

In the Schrodinger picture $\rho$ must be a positive operator at all points. This guarantees that the expectation value of arbitrary positive operators are positive. For instance, $\left\langle\left(A-\langle A\rangle_{\rho}\right)^{2}\right\rangle_{\rho} \geq 0$ (with $A$ Hermitian). And the positivity of $\rho$ must hold at all times, so it ought to be preserved by the dynamical evolution. This is true in the classical and quantum cases, but it is not guaranteed in the hybrid dynamics.

Since we have already seen that the positivity of an observable is not preserved in the hybrid case it follows that the positivity of $\rho$ will also be spoiled. Indeed,

$$
\langle A(t)\rangle_{\rho}=\langle A\rangle_{\rho(t)}
$$

in the Heisenberg and in Schrodinger picture, respectively. If $A$ is positive but $\langle A(t)\rangle_{\rho}$ evolves to negative values (with positive $\rho)$, in the Schrodinger picture this must correspond to a $\rho(t)$ that cannot be positive definite.

We can consider again the example of the particle with classical position-momentum and quantum spin- $\frac{1}{2}$. In that setting a Hamiltonian takes the form

$$
H=h_{c}+\boldsymbol{h}_{q} \cdot \boldsymbol{S}
$$

where $h_{c}$ and $\boldsymbol{h}_{q}$ are $c$-number functions of $(\boldsymbol{x}, \boldsymbol{k})$. The density matrix is

$$
\rho=\alpha+\beta \cdot S
$$

where $\alpha$ and $\boldsymbol{\beta}$ are $c$-number functions of $(\boldsymbol{x}, \boldsymbol{k})$ and $t$, and the positivity condition on $\rho$ is $\alpha \geq \hbar\|\boldsymbol{\beta}\| / 2$.

The dynamical evolution, with the bracket of Eq. 6.24) for $n=2$, takes the form

$$
\frac{d \rho}{d t}=(H, \rho)^{\prime}=\left\{h_{c}, \alpha\right\}+\left\{h_{c}, \boldsymbol{\beta}\right\} \cdot \boldsymbol{S}+\boldsymbol{h}_{q} \times \boldsymbol{\beta} \cdot \boldsymbol{S}+\frac{\hbar^{2}}{4}\left\{\boldsymbol{h}_{q} \cdot \boldsymbol{\beta}\right\} .
$$

That is

$$
\begin{aligned}
& \partial_{t} \alpha=\left\{h_{c}, \alpha\right\}+\frac{\hbar^{2}}{4}\left\{\boldsymbol{h}_{q} \cdot \boldsymbol{\beta}\right\}, \\
& \partial_{t} \boldsymbol{\beta}=\left\{h_{c}, \boldsymbol{\beta}\right\}+\boldsymbol{h}_{q} \times \boldsymbol{\beta} .
\end{aligned}
$$

It is instructive to see how positivity of $\rho$ is preserved in the classical and quantum cases. In the purely classical case $\boldsymbol{h}_{q}=\boldsymbol{\beta}=0$, and the only remaining relation is $\partial_{t} \alpha=\left\{h_{c}, \alpha\right\}$. If $\alpha \geq 0$ ever reaches the value 0 at some point, this must be a minimum, and so $\{, \alpha\}$ vanishes there; this prevents $\alpha$ from passing from positive to negative values. On the other hand in the 
purely quantal case, all functions are $(\boldsymbol{x}, \boldsymbol{k})$-independent, and the non trivial relations are $\alpha=$ constant and $\partial_{t} \boldsymbol{\beta}=\boldsymbol{h}_{q} \times \boldsymbol{\beta}$. This generates a continuous rotation of the vector $\boldsymbol{\beta}$ around $\boldsymbol{h}_{q}$, so if $\hbar\|\boldsymbol{\beta}\| / 2 \leq \alpha$ at $t=0$ it will remain so at all times.

In the hybrid case positivity of $\rho$ is not so protected. Let us consider for instance the simple case of

$$
\boldsymbol{h}_{q}=h_{q} \hat{e}_{z}, \quad \boldsymbol{\beta}=\beta \hat{e}_{z}, \quad(t=0) .
$$

With this dynamics the form of $\boldsymbol{\beta}$ parallel to the $z$ axis is preserved by the evolution. The equations become

$$
\begin{aligned}
& \partial_{t} \alpha=\left\{h_{c}, \alpha\right\}+\frac{\hbar^{2}}{4}\left\{h_{q}, \beta\right\}, \\
& \partial_{t} \beta=\left\{h_{c}, \beta\right\} .
\end{aligned}
$$

Here we see that the positivity condition $\alpha \geq \hbar|\beta| / 2$ at $t=0$ needs not be preserved at later times: any of the functions $\alpha^{\prime} \equiv \alpha \pm \hbar \beta / 2$ fulfills the equation

$$
\partial_{t} \alpha^{\prime}=\left\{h_{c}, \alpha^{\prime}\right\}+\frac{\hbar^{2}}{4}\left\{h_{q}, \beta\right\} .
$$

If $\alpha^{\prime}$ becomes zero at some point $(\boldsymbol{x}, \boldsymbol{k})$, this will be a minimum and $\left\{h_{c}, \alpha^{\prime}\right\}$ will vanish there, however, the other contribution $\frac{\hbar^{2}}{4}\left\{h_{q}, \beta\right\}$ can be negative, since $\beta$ follows its own autonomous dynamics. So nothing prevents from having $\partial_{t} \alpha^{\prime}<0$ at a point where $\alpha^{\prime}=0$. In that case $\alpha^{\prime}$ would become negative there, implying a violation of positivity of $\rho(t)$. Of course, an analysis similar to this one can carried out in the Heisenberg picture to show that positivity of an observable is not preserved.

The explicit solution of $\rho(t)$ in Eq. 6.34) also illustrates that positivity is not preserved in general: The term in $\alpha(t)$ depending linearly with $t$ (while the other terms are bounded) implies that $\alpha(t)$ can become negative.

\section{Discussion}

Clearly, preservation of the positivity of $\rho$ is a condition that must be imposed to any hybrid dynamics. Since our solution is unique using the strong postulates, we have to consider weaker axioms. The condition of preservation of positivity is somewhat more difficult to impose because it is does not have an algebraic form. We do not attempt to work it out here.

We have seen that there is no problem of positivity in the classical or quantum cases and this is also true in the Ehrenfest hybrid formalism discussed at the end of Sec. \C There, the density $\sigma(\boldsymbol{X})$ on $\mathscr{M}$ evolves as $\sigma(\boldsymbol{X} ; t)=\sigma(\boldsymbol{X}(-t))$ and obviously positivity is preserved (the points $\boldsymbol{X}$ move but preserve their weight). The $\rho$ corresponding to a given $\sigma$ is easily obtained from

$$
\langle A\rangle_{\sigma}=\int d x d k d \psi d \psi^{*} \sigma(\boldsymbol{X})\langle A(\boldsymbol{x}, \boldsymbol{k})\rangle_{\psi}=\langle\langle A \rho\rangle\rangle
$$

hence

$$
\rho=\int d \psi d \psi^{*} \sigma(\boldsymbol{X})|\psi\rangle\langle\psi|
$$

Note, incidentally, that there is an enormously larger number of different $\sigma$ than $\rho$. This is a manifestation of the explosion of observables in the Ehrenfest approach. Also, different $\sigma$ producing the same $\rho$ at $t=0$ will evolve producing different $\rho(t)$ at later times. This breaks statistical consistency.

Clearly $\rho$ is positive since $\sigma$ is positive, and this property is preserved by the evolution. We noted at the end of Sec. IIC that our formulation can be cast similarly on $\mathscr{M}$ with the observables being functions of bilinear type, but it cannot be based on evolution from orbits $\boldsymbol{X}(t)$ on $\mathscr{M}$ as this breaks bilinearity. Nevertheless, one observes that the positivity preserving flow of $\boldsymbol{\sigma}(\boldsymbol{X})$ follows a continuity equation $\partial_{t} \sigma=\boldsymbol{\nabla}_{X} \cdot\left(\boldsymbol{v}_{X} \sigma\right)$ while the observables obey the adjoint relation $\partial_{t} F=\boldsymbol{v}_{X} \cdot \nabla_{X} F{ }^{15}$ The conservation properties of the continuity equation depend on the fact that $\boldsymbol{v}_{X} \cdot \nabla_{X}$ is a derivation. If this would translate as is to our formulation, we should require that, in order to preserve $\rho$, the adjoint of $(,)^{\prime}$ should be a derivation, that is $($,$) should$ be a derivation. If this is the case, we can certainly conclude that no solution exists, as we showed above (Sec. IB ) that ( , ) can never be a derivation in $\mathscr{A}$ (even with the weak postulates). However the translation is not completely obvious so we cannot give a final answer at present.

\footnotetext{
${ }^{15}$ In the Hamiltonian case both operators $\boldsymbol{\nabla}_{X} \cdot \boldsymbol{v}_{X}$ and $\boldsymbol{v}_{X} \cdot \boldsymbol{\nabla}_{X}$ coincide due to $\left(\boldsymbol{\nabla}_{X} \cdot \boldsymbol{v}_{X}\right)=0$.
} 


\section{SUMMARY AND CONCLUSIONS}

In this work we have followed the seemingly most natural path to formulating exact quantum-classical hybrid systems. As discussed in Sec. III this entails to have a set of hybrid observables for which we take the tensor product of the classical and the quantum spaces of observables [Eq. [2.5]], in full analogy with the classical-classical or quantum-quantum cases. Next, in the Heisenberg picture we have to provide a dynamical bracket between observables, similar to the Poisson bracket and the commutator, [Eq. [2.7p]. To do so we have introduced conditions to be fulfilled by the bracket. The minimal postulates are those in Eqs. (2.8), (2.9) and (2.12), together with the Lie bracket property, and in particular the Jacobi identity [Eq. (4.11)]. Another essential condition is the preservation of the range of the observable (Sec. VIII). We have not attempted to obtain the most general Lie bracket fulfilling the minimal postulates as that problem looks hard. Instead, we have considered stronger postulates [Eq. [2.24]] for which we have been able to obtain a complete solution in the case of finite-dimensional Hilbert spaces, [Eq. [4.9]]. This complements a previous result in [25] showing that such dynamical bracket does not exists for $\mathscr{H}=L^{2}\left(\mathbb{R}^{n}\right)$. The proof of the Lie bracket property is given in Sec. IVB The bracket is obtained constructively in Sec. $\mathrm{V}$ where it is shown that the solution is unique (with some additional assumptions of universality). Generalizations are discussed in Appendix A The adjoint bracket, which describes the evolution of the density matrix in the Schrodinger picture is explicitly obtained in Sec. VI] The ideas have been illustrated in Secs. [II and VI with the case of a particle with classical position-momentum and quantum spin- $\frac{1}{2}$. The failure to comply with positivity (Sec. VII) is a serious problem which invalidates the solution found. One possible way out would be to weaken the postulates. This would allow new solutions perhaps one of them preserving the positivity of the matrix density. In the same section we have speculated that the preservation of the positivity of the density matrix in its evolution could require $(, H)$ to be a derivation (as in the classical or quantum cases). Since this requisite can never be satisfied in the hybrid case, this would be a no-go situation. Apart from this speculation, there is the real possibility that no acceptable solution exists even for the minimal postulates. In this case, either one has to admit more exotic formulations somehow sorting the many problems noted in Sec. IC or else admit that no formulation of the exact type exists for quantum-classical systems, which would remain as useful approximations of quantum-quantum systems.

\section{Acknowledgments}

I thank M. Hall and M. Reginatto for bringing Ref. [52] to my attention. This work was supported by Spanish Ministerio de Economía y Competitividad and European FEDER funds (grant FIS2014-59386-P), and by the Agencia de Innovación y Desarrollo de Andalucía (grant FQM225).

\section{Appendix A: Mathematical structure of the hybrid bracket}

Here we want to analyze the mathematical structure of our hybrid bracket and its possible generalizations.

In the theory of Lie algebras, the semidirect sum of two Lie algebras is defined as follows [54]. Let $L_{1}$ and $L_{2}$ be two Lie algebras with Lie products denoted by $[,]_{1}$ and $[,]_{2}$, respectively. In addition, for any $X \in L_{2}$, let $D_{X}$ be a derivation on $L_{1}$, that is,

$$
\forall X \in L_{2} \quad \forall Y, Z \in L_{1} \quad D_{X}(Y) \in L_{1}, \quad D_{X}\left([Y, Z]_{1}\right)=\left[D_{X}(Y), Z\right]_{1}+\left[Y, D_{X}(Z)\right]_{1} .
$$

Then, if $X \rightarrow D_{X}$ is a homomorphism, that is,

$$
\forall X, Y \in L_{2}, \quad D_{[X, Y]_{2}}=\left[D_{X}, D_{Y}\right],
$$

one can define a new Lie algebra

$$
L=L_{1} \oplus_{s} L_{2}
$$

called the semidirect sum of $L_{1}$ and $L_{2}$. The vector space of $L$ is the external direct sum of $L_{1}$ and $L_{2}$. External means that the elements of $L$ are ordered pairs of elements $X_{1} \in L_{1}$ and $X_{2} \in L_{2}$ denoted by $X_{1}+X_{2}$. Within $L, L_{1}$ and $L_{2}$ are linearly independent by definition and in the finite dimensional case $\operatorname{dim} L=\operatorname{dim} L_{1}+\operatorname{dim} L_{2}$. The Lie product of two elements of $L$ is defined as

$$
\left[X_{1}+X_{2}, Y_{1}+Y_{2}\right]=\left[X_{1}, Y_{1}\right]_{1}+\left[X_{2}, Y_{2}\right]_{2}+D_{X_{2}}\left(Y_{1}\right)-D_{Y_{2}}\left(X_{1}\right),
$$

where $X_{1}, Y_{1} \in L_{1}$ and $X_{2}, Y_{2} \in L_{2}$. It is readily verified that this is a Lie bracket using Eqs. A1 and A2 .

In the hybrid quantum-classical case, $L_{2}$ is $\mathscr{A}_{c}$, understood as a set of classical observables endowed with the Poisson bracket as Lie product. This set needs not be all classical observables, but it must be a Lie algebra, i.e., a linear space closed under the Poisson bracket

$$
\left\{\mathscr{A}_{c}, \mathscr{A}_{c}\right\} \subseteq \mathscr{A}_{c}
$$


Further, $L_{1}$ is $\tilde{\mathscr{A}}$ understood as a set of hybrid observables (functions on the phase space taking values in operators) with the requirement of being a Lie algebra with the commutator, that is, a linear space such that

$$
\llbracket \tilde{\mathscr{A}}, \tilde{\mathscr{A}} \rrbracket \subseteq \tilde{\mathscr{A}} .
$$

So here we no longer insist on the Hilbert space being finite dimensional and the observables of $\tilde{\mathscr{A}}$ being traceless. Also, $\tilde{\mathscr{A}}$ needs not be closed under multiplication. For the derivation $D_{X}$ we take the Poisson bracket, $D_{X}=\{X$, $\}$, since it fulfills Eqs. (A1) and A2). Because $D_{X}(Y) \in L_{2}$, we further require

$$
\left\{\mathscr{A}_{c}, \tilde{\mathscr{A}}\right\} \subseteq \tilde{\mathscr{A}}
$$

Under these conditions we can construct the semidirect sum

$$
\mathscr{A}=\tilde{\mathscr{A}} \oplus_{s} \mathscr{A}_{c},
$$

with

$$
\left(\tilde{A}+C, \tilde{A}^{\prime}+C^{\prime}\right)=\llbracket \tilde{A}, \tilde{A}^{\prime} \rrbracket+\left\{C, C^{\prime}\right\}+\left\{C, \tilde{A}^{\prime}\right\}-\left\{C^{\prime}, \tilde{A}\right\}
$$

which will be a Lie bracket in $\mathscr{A}$.

One way to guarantee Eqs. A5, A6 and (A7), is to include in $\mathscr{A}_{c}$ all classical observables and take $\tilde{\mathscr{A}}^{\mathrm{A}}=\mathscr{A}_{c} \otimes \tilde{\mathscr{A}}_{q}$, where

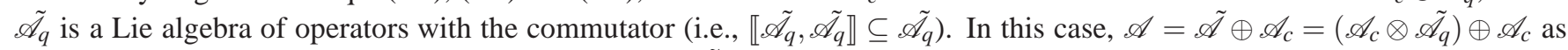
vector space, or equivalently $\mathscr{A}=\mathscr{A}_{c} \otimes \mathscr{A}_{q}$ with $\mathscr{A}_{q}=\tilde{\mathscr{A}}_{q} \oplus U$, where $U$ is a one dimensional vector space.

In the particular case in which the identity of the Hilbert space is not contained in $\tilde{\mathscr{A}}_{q}$, one can simply identify $U$ with $\{\lambda \mathbb{1}, \lambda \in \mathbb{R}\}$, and $\tilde{A}+C$ with $\tilde{A}+C \mathbb{1}$. This is what has been done in Sec. IV for a finite dimensional Hilbert, along with choice of $\tilde{\mathscr{A}}_{q}$ as the set of traceless operators.

For $\mathscr{H}=L^{2}\left(\mathbb{R}^{n}\right)$, the condition $\llbracket \tilde{\mathscr{A}}_{q}, \tilde{\mathscr{A}}_{q} \rrbracket \subseteq \tilde{\mathscr{A}}_{q}$ implies that $\mathbb{1} \in \tilde{\mathscr{A}}_{q}$ (at least for $\tilde{\mathscr{A}}_{q}$ sufficiently large to be useful). So, if one insists in carrying out the construction above, one would have $\mathbb{1}$ in $\tilde{\mathscr{A}}_{q}$ and an additional, linearly independent $\mathbb{1}^{\prime}$ in $U$. The resulting $\mathscr{A}$, or more specifically $\mathscr{A}_{q}=\tilde{\mathscr{A}}_{q} \oplus U$, does not conform to the structure assumed in Sec. ЏA, since $\tilde{\mathscr{A}}_{q}$ would be all quantum operators and $\mathscr{A}_{q}$ would be something else, with a new dimension along $\mathbb{1}^{\prime}$ outside the original Hilbert space and with no obvious physical meaning.

Another observation is that in the finite dimensional case, one can choose some Lie algebra of operators $\tilde{\mathscr{A}}_{q}$ not including $\mathbb{1}$ and make the construction with $\mathscr{A}_{q}=\tilde{\mathscr{A}}_{q} \oplus U$ (with $U=\{\lambda \mathbb{1}, \lambda \in \mathbb{R}\}$ ) and $\mathscr{A}=\mathscr{A}_{c} \otimes \mathscr{A}_{q}$, as already noted. Often, for a given space $\mathscr{A}_{q}$, the requirement of $\tilde{\mathscr{A}}_{q}$ being a Lie algebra will completely fix this space in the decomposition $\mathscr{A}_{q}=\tilde{\mathscr{A}}_{q} \oplus U$ [always with $U=\{\lambda \mathbb{1}, \lambda \in \mathbb{R}\}]$, but not in all cases. In those cases, each choice of $\tilde{\mathscr{A}}_{q}$ will define a different hybrid bracket. So the proof of uniqueness in Sec. $\mathrm{V}$ relies on the assumption that all operators are included in $\mathscr{A}_{q}$. This assumption is also used explicitly in the construction of the hybrid bracket in the Schrodinger picture, $(,)^{\prime}$.

\section{Appendix B: Proof of Eq. 5.19}

In this Appendix we proof that Eqs (5.14) and 5.18) imply Eq. 5.19). Since, Eq. 5.18 is identical for the three brackets $(,)_{\delta},(,)_{f}$ and $(,)_{d}$, we just consider one of them explicitly.

We explicitly assume that our classical sector is of position-momentum type, with $\mathscr{A}_{c}$ containing all functions of $(\boldsymbol{x}, \boldsymbol{k})$. It is not clear whether the proof covers the case of restricted subsets of classical observables (but still forming a Lie algebra, $\left\{\mathscr{A}_{c}, \mathscr{A}_{c}\right\} \subseteq \mathscr{A}_{c}$ ) as in Eq. (2.3).

Following [25], let us introduce a plane-wave basis in the set of functions defined on the phase space

$$
e_{r}=\exp \left(i \boldsymbol{k}_{r} \cdot \boldsymbol{x}-i \boldsymbol{x}_{r} \cdot \boldsymbol{k}\right), \quad \boldsymbol{x}_{r}, \boldsymbol{k}_{r} \in \mathbb{R}^{n} .
$$

$(\boldsymbol{x}, \boldsymbol{k})$ are the classical dynamical variables and $\boldsymbol{x}_{r}, \boldsymbol{k}_{r}$ parameters. These basis functions fulfill the relations

$$
\left\{e_{r}, \boldsymbol{x}\right\}=i \boldsymbol{x}_{r} e_{r}, \quad\left\{e_{r}, \boldsymbol{k}\right\}=i \boldsymbol{k}_{r} e_{r},
$$

and also

$$
\left\{e_{r}, e_{s}\right\}=v_{r s} e_{r+s}
$$

with

$$
v_{r s} \equiv \boldsymbol{k}_{r} \cdot \boldsymbol{x}_{s}-\boldsymbol{x}_{r} \cdot \boldsymbol{k}_{s}, \quad\left(\boldsymbol{x}_{r+s}, \boldsymbol{k}_{r+s}\right) \equiv\left(\boldsymbol{x}_{r}+\boldsymbol{x}_{s}, \boldsymbol{k}_{r}+\boldsymbol{k}_{s}\right) .
$$


Using this basis

$$
\left(e_{r}, e_{s}\right)_{\delta}=\sum_{t} F_{r s t} e_{t}
$$

for some function $F_{r s t}$. However, using Eqs. (5.18) and (B2),

$$
\left\{\left(e_{r}, e_{s}\right)_{\delta}, \boldsymbol{x}\right\}=i \boldsymbol{x}_{r+s}\left(e_{r}, e_{s}\right)_{\delta}, \quad\left\{\left(e_{r}, e_{s}\right)_{\delta}, \boldsymbol{k}\right\}=i \boldsymbol{k}_{r+s}\left(e_{r}, e_{s}\right)_{\delta}
$$

This implies [comparing with Eq. (B2)] that in the sum over $t$ in Eq. (B5), only the term $t=r+s$ can have a contribution. That is,

$$
\left(e_{r}, e_{s}\right)_{\delta}=F_{r s} e_{r+s}
$$

where $F_{r s}$ is some function of $\left(\boldsymbol{x}_{r}, \boldsymbol{k}_{r}\right)$ and $\left(\boldsymbol{x}_{s}, \boldsymbol{k}_{s}\right)$.

In order to pinpoint this function, let us impose Eq. (5.18) with $C=e_{r}, C^{\prime}=e_{s}$ and $C^{\prime \prime}=e_{t}$. This gives

$$
F_{r s} v_{r+s, t}=v_{r t} F_{r+t, s}+v_{s t} F_{r, s+t} \quad \forall r, s, t .
$$

Let us show that this relation implies that $F_{r s}$ must be a function of $v_{r s}$ only. ${ }^{16}$ To do this let us take

$$
\boldsymbol{k}_{t}=-\boldsymbol{k}_{r}, \quad \boldsymbol{x}_{t}=-\boldsymbol{x}_{r}-v_{r s} \frac{\boldsymbol{k}_{s}}{\boldsymbol{k}_{s}^{2}} .
$$

With this choice

$$
v_{s t}=0, \quad v_{r+s, t}=v_{r t} .
$$

Hence $F_{r s}=F_{r+t, s}$, or more explicitly

$$
F\left(\boldsymbol{x}_{r}, \boldsymbol{k}_{r} ; \boldsymbol{x}_{s}, \boldsymbol{k}_{s}\right)=F\left(-v_{r s} \frac{\boldsymbol{k}_{s}}{\boldsymbol{k}_{s}^{2}}, \mathbf{0} ; \boldsymbol{x}_{s}, \boldsymbol{k}_{s}\right) .
$$

That is, for given $\left(\boldsymbol{x}_{s}, \boldsymbol{k}_{s}\right)$, all the dependence on $\left(\boldsymbol{x}_{r}, \boldsymbol{k}_{r}\right)$ is through $v_{r s}$. Taking an analogous choice of $t$ this time with $v_{r t}=0$, one obtains that all dependence on $\left(\boldsymbol{x}_{s}, \boldsymbol{k}_{s}\right)$ is also through $v_{r s}$. So

$$
F_{r s}=f\left(v_{r s}\right)
$$

for some function $f(v)$ to be specified, and Eq. (B8) becomes

$$
f\left(v_{r s}\right) v_{r+s, t}=v_{r t} f\left(v_{r+t, s}\right)+v_{s t} f\left(v_{r, s+t}\right) .
$$

Taking

$$
v_{1} \equiv v_{r s}, \quad v_{2} \equiv v_{r+t, s}, \quad v_{3} \equiv v_{r, s+t},
$$

as independent variables, so that

$$
v_{r+s, t}=v_{3}-v_{2}, \quad v_{r t}=v_{3}-v_{1}, \quad v_{s t}=v_{1}-v_{2},
$$

the equation on $f(v)$ becomes

$$
f\left(v_{1}\right)\left(v_{3}-v_{2}\right)=\left(v_{3}-v_{1}\right) f\left(v_{2}\right)+\left(v_{1}-v_{2}\right) f\left(v_{3}\right),
$$

where $v_{1}, v_{2}, v_{3}$ are arbitrary. Paying attention to any of the three variables with the other two fixed, it follows that

$$
f(v)=\alpha v+\beta
$$

\footnotetext{
${ }^{16}$ The idea is that if $t$ is such that $v_{s t}=0$ then $v_{r+s, t}=v_{r t}$ and hence $F_{r s}=F_{r+t, s}$. That is, $r$, which is a $2 n$-dimensional variable, can be changed in $2 n-1$ directions (those of $t$ restricted by $v_{s t}=0$ ) without modifying $F_{r s}$, so the dependence on $r$ is really through $v_{r s}$.
} 
for some constants $\alpha$ and $\beta$. In turn this forms fulfills Eq. $\mathrm{B} 16$ identically. Hence the most general solution is

$$
\left(e_{r}, e_{s}\right)_{\delta}=\left(\alpha v_{r s}+\beta\right) e_{r+s}
$$

which corresponds to the bracket

$$
\left(C, C^{\prime}\right)_{\delta}=\alpha\left\{C, C^{\prime}\right\}+\beta C C^{\prime}
$$

Since $(,)_{\delta}$ and $(,)_{d}$ are antisymmetric, $\beta=0$ in those cases. On the other hand $(,)_{f}$ is symmetric, so $\alpha=0$ for that bracket. This proves Eq. 5.19].

[1] N. Bohr, Atomic Physics and Human Knowledge (Wiley, New York, 1958).

[2] W. Heisenberg, Physics and Philosophy: The Revolution in Modern Science (Harper Perennial Modern Classics, London, 2007).

[3] B. dEspagnat, Conceptual Foundations of Quantum Mechanics (Addison Wesley, Reading, 1976).

[4] A. E. Allahverdyan, R. Balian and T. M. Nieuwenhuizen, "Understanding quantum measurement from the solution of dynamical models," Phys. Rept. 525, 1 (2013) doi:10.1016/j.physrep.2012.11.001 [arXiv:1107.2138 [quant-ph]].

[5] L. Rosenfeld, "On quantization of fields," Nucl. Phys. 40 (1963) 353. doi:10.1016/0029-5582(63)90279-7

[6] T. W. B. Kibble and S. Randjbar-Daemi, "Nonlinear Coupling of Quantum Theory and Classical Gravity," J. Phys. A 13, 141 (1980). doi:10.1088/0305-4470/13/1/015

[7] C. J. Isham, in Quantum Gravity: An Overview, Quantum Gravity 2: a Second Oxford Symposium, edited by C. J. Isham, R. Penrose, and D. W. Sciama (Oxford University Press, Oxford, 1981), pp. 162.

[8] D. N. Page and C. D. Geilker, "Indirect Evidence for Quantum Gravity," Phys. Rev. Lett. 47, 979 (1981). doi:10.1103/PhysRevLett.47.979

[9] E. Alvarez, "Quantum Gravity: A Pedagogical Introduction To Some Recent Results," Rev. Mod. Phys. 61, 561 (1989). doi:10.1103/RevModPhys.61.561

[10] S. Boughn, "Nonquantum Gravity," Found. Phys. 39, 331 (2009). doi:10.1007/s10701-009-9282-0 [arXiv:0809.4218 [gr-qc]].

[11] S. Carlip, "Is Quantum Gravity Necessary?," Class. Quant. Grav. 25, 154010 (2008). doi:10.1088/0264-9381/25/15/154010 [arXiv:0803.3456 [gr-qc]].

[12] M. Derakhshani, "Newtonian Semiclassical Gravity in the Ghirardi-Rimini-Weber Theory with Matter Density Ontology,” Phys. Lett. A 378, 990 (2014) doi:10.1016/j.physleta.2014.02.005 [arXiv:1304.0471] [gr-qc]].

[13] J. Åqvist and A. Warshel, "Simulation of enzyme reactions using valence bond force fields and other hybrid quantum/classical approaches," Chem. Rev. 93, 2523 (1993). doi:10.1021/cr00023a010

[14] G. Monard, M. Loos, V. Théry, K. Baka, and J-L. Rivail, "Hybrid classical quantum force field for modeling very large molecules," Int. J. Quantum Chem. 58, 153 (1996). doi:10.1002/(SICI)1097-461X(1996)58:2<153::AID-QUA4>3.0.CO;2-X

[15] F. A. Bornemann, P. Nettesheim, and C. Schütte "Quantumclassical molecular dynamics as an approximation to full quantum dynamics" J. Chem. Phys. 105, 1074 (1996). doi:10.1063/1.471952

[16] O. V. Prezhdo and P. J. Rossky, "Mean-field molecular dynamics with surface hopping," J. Chem. Phys. 107, 825 (1997). doi:10.1063/1.474382

[17] R. Kapral and G. Ciccotti, "Mixed quantum-classical dynamics,” J. Chem. Phys. 110, 8919 (1999). doi:10.1063/1.478811

[18] G. Csányi, T. Albaret, M. C. Payne and A. De Vita, "Learn on the Fly: A Hybrid Classical and Quantum-Mechanical Molecular Dynamics Simulation,” Phys. Rev. Lett. 93, 175503 (2004). doi:10.1103/PhysRevLett.93.175503

[19] W. Magnus, "On the exponential solution of differential equations for a linear operator," Commun. Pure Appl. Math. 7, 649 (1954). doi:10.1002/cpa.3160070404

[20] S. Blanes, F. Casas, J. A. Oteo, J. Ros, “The Magnus expansion and some of its applications," Phys. Rep. 470, 151 (2009). doi:10.1016/j.physrep.2008.11.001

[21] C. Barcelo, R. Carballo-Rubio, L. J. Garay and R. Gomez-Escalante, "Hybrid classical-quantum formulations ask for hybrid notions," Phys. Rev. A 86, 042120 (2012) doi:10.1103/PhysRevA.86.042120 [arXiv:1206.7036 [quant-ph]].

[22] I. V. Aleksandrov, "The statistical dynamics of a system consisting of a classical and a quantum subsystem," Z. Naturforsch. 36A, 902 (1981).

[23] W. Boucher and J. H. Traschen, "Semiclassical Physics and Quantum Fluctuations," Phys. Rev. D 37, 3522 (1988). doi:10.1103/PhysRevD.37.3522

[24] A. Anderson, "Quantum back reaction on 'classical' variables," Phys. Rev. Lett. 74, 621 (1995). doi:10.1103/PhysRevLett.74.621 [hep-th/9406182].

[25] J. Caro and L. L. Salcedo, "Impediments to mixing classical and quantum dynamics," Phys. Rev. A 60, 842 (1999) doi:10.1103/PhysRevA.60.842 |quant-ph/9812046|.

[26] H. Goldstein, C. Poole, and J. Safko, Classical Mechanics, 3rd ed. (Addison Wesley, New York, 2002).

[27] T. A. Oliynyk, "Classical-Quantum Limits," Found. Phys. 46, no. 12, 1551 (2016) doi:10.1007/s10701-016-0028-5 [arXiv:1508.04477 [quant-ph]].

[28] O. V. Prezhdo, "A quantum-classical bracket that satisfies the Jacobi identity," J. Chem. Phys. 124, 201104 (2006). doi:10.1063/1.2200342

[29] L. L. Salcedo, "Comment on 'A quantum-classical bracket that satisfies the Jacobi identity' [J.Chem.Phys.124,201104 (2006)]," J. Chem. Phys. 126, 057101 (2007) doi:10.1063/1.2431650 [quant-ph/0701054]. 
[30] O. V. Prezhdo, "Reply to Comment on A quantum-classical bracket that satisfies the Jacobi identity [J. Chem. Phys.124, 201104 (2006)]," J. Chem. Phys. 126, 057102 (2007). doi:10.1063/1.2431651

[31] J. Schwinger, "Particles, sources, and fields. Volume 1," Reading, Mass., 1970, 425p.

[32] L. L. Salcedo, "Absence of classical and quantum mixing," Phys. Rev. A 54, 3657 (1996) doi:10.1103/PhysRevA.54.3657 [hep-th/9509089].

[33] D. Sahoo, “Mixing quantum and classical mechanics and uniqueness of Planck's constant," J. Phys. A 37, 997 (2004). doi:10.1088/03054470/37/3/031

[34] T. Dass, "A Stepwise Planned Approach to the Solution of Hilbert's Sixth Problem. I : Noncommutative Symplectic Geometry and Hamiltonian Mechanics," arXiv:0909.4606 [math-ph].

[35] J. W. Negele and H. Orland, "Quantum Many Particle Systems," Redwood City, USA: Addison-Wesley (1988) 459 P. (Frontiers in Physics, 68)

[36] L. Diosi, N. Gisin and W. T. Strunz, "Quantum approach to coupling classical and quantum dynamics,” Phys. Rev. A 61, 22108 (2000) doi:10.1103/PhysRevA.61.22108, 10.1103/PhysRevA.61.022108 [quant-ph/9902069].

[37] C. F. Craig, W. R. Duncan and O. V. Prezhdo, "Trajectory Surface Hopping in the Time-Dependent Kohn-Sham Approach for ElectronNuclear Dynamics,” Phys.Rev.Lett. 95, 163001 (2005). doi:10.1103/PhysRevLett.95.163001

[38] Qi Zhang and Biao Wu, "General Approach to Quantum-Classical Hybrid Systems and Geometric Forces," Phys. Rev. Lett. 97, 190401 (2006) doi:10.1103/PhysRevLett.97.190401

[39] J. L. Alonso, A. Castro, J. Clemente-Gallardo, J. C. Cuchi, P. Echenique and F. Falceto, "Statistics and Nosé formalism for Ehrenfest dynamics," J. Math. Phys. 44, 395004 (2011). doi:10.1088/1751-8113/44/39/395004

[40] H. T. Elze, "Linear dynamics of quantum-classical hybrids," Phys. Rev. A 85, 052109 (2012) doi:10.1103/PhysRevA.85.052109 [arXiv:1111.2276 [quant-ph]].

[41] L. Diosi and J. J. Halliwell, "Coupling classical and quantum variables using continuous quantum measurement theory," Phys. Rev. Lett. 81, 2846 (1998) doi:10.1103/PhysRevLett.81.2846 [quant-ph/9705008].

[42] L. L. Salcedo, "Statistical consistency of quantum-classical hybrids," Phys. Rev. A 85, 022127 (2012) doi:10.1103/PhysRevA.85.022127 arXiv:1201.4237[quant-ph]].

[43] M. J. W. Hall and M. Reginatto, "Interacting classical and quantum ensembles," Phys. Rev. A 72, 062109 (2005) doi:10.1103/PhysRevA.72.062109 [quant-ph/0509134].

[44] M. J. W. Hall, “Consistent classical and quantum mixed dynamics,” Phys. Rev. A 78, 042104 (2008). doi:10.1103/PhysRevA.78.042104

[45] G. Sudarshan, "Interaction between classical and quantum systems and the measurement of quantum observables," Pramana 6, 117 (1976). doi:10.1007/BF02847120

[46] D. Chruściński, A. Kossakowski, G. Marmo and E. C. G. Sudarshan, "Dynamics of Interacting Classical and Quantum Systems," Open Syst. Inf. Dyn. 18, 339 (2011). doi:10.1142/S1230161211000236

[47] O. V. Prezhdo and C. Brooksby, "Quantum Backreaction through the Bohmian Particle," Phys. Rev. Lett. 86, 3215 (2001). doi:10.1103/PhysRevLett.86.3215

[48] L. L. Salcedo, "Comment on 'Quantum back reaction through the Bohmian particle'," Phys. Rev. Lett. 90, 118901 (2003). doi:10.1103/PhysRevLett.90.118901

[49] O. V. Prezhdo and C. Brooksby, "Prezhdo and Brooksby Reply:," Phys. Rev. Lett. 90, 118902 (2003). doi:10.1103/PhysRevLett.90.118902

[50] M. Radonjić, S. Prvanović, and N. Burić, "Hybrid quantum-classical models as constrained quantum systems" Phys. Rev. A 85, 064101 (2012). doi:10.1103/PhysRevA.85.064101

[51] A. Peres and D. R. Terno, "Hybrid classical-quantum dynamics," Phys. Rev. A 63, 022101 (2001). doi:10.1103/PhysRevA.63.022101

[52] R. Schiller, "Quasi-Classical Theory of the Spinning Electron,” Phys. Rev. 125, 1116 (1962). doi:10.1103/PhysRev.125.1116

[53] P. Pascual and R. Tarrach, "Qcd: Renormalization For The Practitioner," Lect. Notes Phys. 194, 1 (1984).

[54] A. O. Barut and R. Raczka, “Theory Of Group Representations And Applications,” Singapore, Singapore: World Scientific ( 1986) 717p.

[55] A. Ahmadzadegan, R. B. Mann, D. R. Terno, "How classical is a quantum oscillator?," Phys. Rev. A 93, 032122 (2016). doi:10.1103/PhysRevA.93.032122 